\title{
Computational Aspects of the Helly Property: a Survey
}

\author{
Mitre C. Dourado \\ Núcleo de Computação Eletrônica \\ mitre@nce.ufrj.br
}

\author{
Fábio Protti * \\ Instituto de Matemática and NCE \\ fabiop@nce.ufrj.br
}

\author{
Jayme L. Szwarcfiter * \\ Instituto de Matemática, NCE \\ and COPPE \\ jayme@nce.ufrj.br
}

Universidade Federal do Rio de Janeiro

Caixa Postal 2324, Rio de Janeiro, RJ, Brasil

\begin{abstract}
In 1923, Eduard Helly published his celebrated theorem, which originated the well known Helly property. Say that a family of subsets has the Helly property when every subfamily of it, formed by pairwise intersecting subsets, contains a common element. There are many generalizations of this property which are relevant to some parts of mathematics and several applications in computer science. In this work, we survey computational aspects of the Helly property. The main focus is algorithmic. That is, we describe algorithms for solving different problems arising from the basic Helly property. We also discuss the complexity of these problems, some of them leading to $N P$-hardness results.
\end{abstract}

Keywords: Computational Complexity, Helly property, NP-complete problems.

\section{Introduction}

In 1923, Eduard Helly [24, 57] published the famous theorem which originated the so called Helly property. The theorem asserts that in a $d$-dimensional euclidian space, if in a finite collection of $n>d$ convex sets any $d+1$ sets have a point in common, then there is a point in common to all sets. This theorem has been extensively studied in distinct parts of mathematics and other areas, as computer science. In fact, it has a central role in the studies of geometric transversal theory, combinatorial geometry and convexity theory.

*Partially supported by CNPq and FAPERJ.
A few surveys have been written on the Helly property. We mention [27, 43, 51]. The Helly property has been the object of studies in extremal hypergraph theory, as [87], and in other topics of the study of graphs. For instance, see $[39,88,89]$. There are many extensions of the Helly property. One of the generalizations, the fractional Helly property, is directly related to Alon and Kleitman's result [4], solving a famous conjecture by Hadwiger and Debrunner.

Besides the purely theoretical interest, the Helly property has applications to some different areas. For example, in the context of optimization, it has been applied to location problems [36], and generalized linear programming [5]. In computer science, the Helly property has been used in the theory of semantics [10], coding [9], computational biology [79], data bases [45, 46], image processing [22] and clearly graphs and hypergraphs.

In this work, we survey some of the results on the Helly property, from the computational point of view. Our purpose is to describe algorithms and complexity results for many structural algorithmic problems, related to the Helly property and some of its generalizations. In addition, we also include some new proposals of algorithms, for some specific problems. Besides describing the algorithms and complexity for the considered problems, we also formulate the main structural characterizations, which are the basis of the algorithms.

Following, we give some definitions and notation used throughout this paper.

A hypergraph $\mathcal{H}$ is an ordered pair $(V(\mathcal{H}), E(\mathcal{H}))$ where $V(\mathcal{H})=\left\{v_{1}, \ldots, v_{n}\right\}$ is a finite set of vertices and $E(\mathcal{H})=\left\{E_{1}, \ldots, E_{m}\right\}$ is a set of nonempty hyper- 
edges $E_{i} \subseteq V(\mathcal{H})$. When there is no ambiguity we will denote the number of vertices and of hyperedges of a hypergraph $\mathcal{H}$ by $n$ and $m$, respectively. Since the Helly property and most variations considered in this work deal with the hyperedges of a hypergraph, isolated vertices are not relevant, and can be dropped. Hence, unless otherwise stated, we assume in all the text that for a hypergraph $\mathcal{H}$, $V(\mathcal{H})=\bigcup_{E_{i} \in E(\mathcal{H})} E_{i}$.

Let $\mathcal{H}$ be a hypergraph. We say that $\mathcal{H}$ is a $k$ hypergraph if $|E(\mathcal{H})|=k$; a $k^{-}$-hypergraph if $|E(\mathcal{H})| \leq$ $k$; and a $k^{+}$-hypergraph if $|E(\mathcal{H})| \geq k$. We use the same notation for a term standing for a set, for example, given a set $S$ with $k$ elements, we can say that $S$ is a $k$-set, or a $(k-1)^{+}$-set, and so on.

The rank $r(\mathcal{H})$ of a hypergraph $\mathcal{H}$ is the maximum cardinality among the hyperedges of $\mathcal{H}$. A hypergraph $\mathcal{H}^{\prime}$ is a partial hypergraph of $\mathcal{H}$ if $E\left(\mathcal{H}^{\prime}\right) \subseteq E(\mathcal{H})$; and $\mathcal{H}^{\prime}$ is a subhypergraph of $\mathcal{H}$ induced by $V^{\prime} \subseteq V(\mathcal{H})$ if $\mathcal{H}^{\prime}$ contains exactly the hyperedges $E_{i} \cap V^{\prime} \neq \varnothing$, for $1 \leq i \leq m$.

The core of $\mathcal{H}$ is defined as $\operatorname{core}(\mathcal{H})=E_{1} \cap E_{2} \cap$ $\ldots \cap E_{m}$. We say that $\mathcal{H}$ is $(p, q)$-intersecting if every partial $p^{-}$-hypergraph of $\mathcal{H}$ has a $q^{+}$-core. We employ the terms intersecting and $p$-intersecting meaning $(2,1)$ intersecting and $(p, 1)$-intersecting hypergraphs, respectively.

Two hypergraphs $\mathcal{H}, \mathcal{H}^{\prime}$ are isomorphic if there exists a bijection $f: V(\mathcal{H}) \rightarrow V\left(\mathcal{H}^{\prime}\right)$ such that:

$\left\{v_{1}, \ldots, v_{p}\right\} \in E(\mathcal{H}) \Longleftrightarrow\left\{f\left(v_{1}\right), \ldots, f\left(v_{p}\right)\right\} \in E\left(\mathcal{H}^{\prime}\right)$.

Given a hypergraph $\mathcal{H}$, we construct the dual hypergraph $\mathcal{H}^{*}$ of $\mathcal{H}$ creating one vertex $e_{j}$ in $V\left(\mathcal{H}^{*}\right)$ for each hyperedge $E_{j} \in E(\mathcal{H})$; and one hyperedge $A_{i}$ in $E\left(\mathcal{H}^{*}\right)$ for every vertex $a_{i} \in V(\mathcal{H})$, defined as $A_{i}=\left\{e_{j}: a_{i} \in\right.$ $\left.E_{j}\right\}$.

A hypergraph $\mathcal{H}$ is $r$-uniform when every hyperedge of $\mathcal{H}$ contains exactly $r$ vertices. Let $r, n$ be integers, $1 \leq$ $r \leq n$. We define the $r$-complete hypergraph $K_{n}^{r}$ to be a hypergraph consisting of all the $r$-subsets of an $n$-set.

A graph is a 2-uniform hypergraph. Usually, a graph is denoted by $G$. A hyperedge and a partial hypergraph of a graph $G$ are respectively called edge and subgraph of $G$. A spanning subgraph of $G$ is a subgraph with vertex set $V(G)$, and the subgraph of $G$ induced by $V^{\prime}, G\left[V^{\prime}\right]$, is the maximal subgraph of $G$ with vertex set $V^{\prime}$. Two vertices $u$ and $v$ forming an edge of $G$ are adjacent vertices or neighbors in $G$, and we denote such edge by $u v$. The open neighborhood of a vertex $v, N(v)$, is the set formed by the neighbors of $v$; the closed neighborhood of $v$ is $N[v]=N(v) \cup\{v\}$; the disk of radius $k$ is the set of vertices whose distance to $v$ is not lerger than $k$. A vertex $v$ is universal in $G$ if $N[v]=V(G)$.

A path is a sequence of distinct vertices $v_{1}, \ldots, v_{q}$, $q \geq 1$, such that $v_{i} v_{i+1} \in E(G)$, for $1 \leq i \leq q-1$. If, furthermore, $q \geq 3$ and there exists the edge $v_{q} v_{1}$, this sequence is a cycle. A chord of a cycle $C$ is any edge joining two non-consecutive vertices in $C$. The distance between two vertices is the number of edges of a minimum path joining them.

A complete set (independent set) is a subset of pairwise adjacent (nonadjacent) vertices. A bipartite set is a subset $B \subseteq V(G)$, which can be partitioned into $B=V_{1} \cup V_{2}$, where $V_{1}, V_{2}$ are nonempty independent sets. If every $v_{i} \in V_{1}$ and $v_{j} \in V_{2}$ are adjacent, then $B$ is a complete bipartite set. A clique of $G$ is a maximal complete set; and a biclique is a maximal complete bipartite set. A (complete) bipartite graph is a graph induced by a (complete) bipartite set. A graph is $K_{r}$-free if it does not contain $r$-complete sets as subgraphs.

A graph is a tree if there exists exactly one path between every pair of vertices of it. If every cycle with at least 4 vertices has a chord, then $G$ is chordal. The complement of a graph $G$, denoted $\bar{G}$, has $V(G)$ as vertex set, and $u v \in E(\bar{G}) \Longleftrightarrow u v \notin E(G)$. A graph is perfect if it does not contain an odd cycle or a complement of an odd cycle, with at least 5 vertices, as an induced subgraph.

The clique hypergraph of $G, \mathcal{C}(G)$, is the hypergraph formed by the cliques of $G$. Given a hypergraph $\mathcal{H}$, the intersection graph, or line graph, of $\mathcal{H}$ is the graph containing one vertex for every hyperedge of $\mathcal{H}$, and two vertices are adjacent if the corresponding hyperedges intersect. The clique graph $K(G)$ of $G$ is the intersection graph of the clique hypergraph of $G$. The $i$-th iterated clique graph of $G$, denoted $K^{i}(G)$, is defined as follows: $K^{0}(G)=G$, while $K^{i}(G)=K\left(K^{i-1}(G)\right), i \geq 1$.

The contents of this survey is as follows. Section 2 presents the basic Helly property on hypergraphs, with the description of some classical families of hypergraphs. A test for the Helly property on hypergraphs is also included. Section 3 also discusses the basic Helly property, now for graphs. The commonest Helly classes of graphs are described, together with their characterizations and recognition algorithms. Section 4 considers the $p$-Helly hypergraphs and a generalization of them, the list $p$-Helly hypergraphs. Section 5 considers the Helly property on subfamilies of limited size. That is, when the cardinality of the subfamilies to be checked for a common vertex is bounded by a positive $k$. Section 6 contains a generalization of the $p$-Helly property which considers the cardinality of the intersections, the $(p, q, s)$-Helly property. Characterizations generalizing classical results on $p$-Helly hy- 
pergraphs and conformal hypergraphs are given; the last one is a new result. This concept is also used to generalize the Helly number of a hypergraph. In Section 7 we apply the $(p, q, s)$-Helly property to graphs. Characterization and recognition of $(p, q)$-clique-Helly graphs are presented. Also the complexity of determining the Helly defect of a graph is discussed. In Section 8 we consider the hereditary Helly property applied to special families of vertices of a graph, such as cliques, disks, bicliques, open and closed neighborhoods. Furthermore we characterize the hereditary $p$-Helly property on graphs and hypegraphs. Section 9 contains a summary of the computational aspects of the problems related in this work. In the last section we list some proposed problems.

\section{Basic Helly Property on Hypergraphs}

In this section, we discuss the basic Helly property on hypergraphs. First, we describe some classical examples of special families of objects satisfying the Helly property. Afterwards, we consider general Helly hypergraphs, and give an algorithm for recognizing this class. Further, we describe some well known classes of hypergraphs where the Helly property holds.

Relevant general references for this section are [11, $12,14,21,38,70]$.

\subsection{General hypergraphs}

A hypergraph is Helly when every intersecting partial hypergraph of it has a nonempty core. For example, the hypergraph $\mathcal{H}$, having $V(\mathcal{H})=\{1,2,3,4\}$ and $E(\mathcal{H})=\{\{1,2\},\{1,3\},\{1,4\}\}$ is Helly, while if $E(\mathcal{H})=\{\{1,2\},\{1,3\},\{2,3\}\}$ then $\mathcal{H}$ is not Helly.

Some classical examples of objects satisfying the Helly property are the following. Intervals of a straight line form a Helly family, as it can be easily observed. Another classical example, known as the Chinese Theorem, expresses a property of arithmetic expressions: let $\mathcal{H}$ be the hypergraph having the integers as vertices, and the arithmetic expressions formed by those integers as hyperedges. Then $\mathcal{H}$ is Helly. Another commonly employed case of a Helly family is the family of subtrees of a tree. The fact that subtrees of a tree are Helly is the basis for many properties of chordal graphs.

From the computational point of view, a central question is to describe a method for recognizing Helly hypergraphs. Observe that simply applying the definition would not lead to an efficient method, since the number of intersecting partial hypergraphs could be exponential in the number of vertices.
Problem 2.1 (Helly HyPERGRAPH): Given a hypergraph $\mathcal{H}$, decide whether $\mathcal{H}$ satisfies the Helly property.

The following algorithm [11] decides if a given hypergraph $\mathcal{H}$ is Helly.

Algorithm 2.1 [11] (RECOGNIZING HELLY HYPER-
GRAPHS): For every triple $T$ of vertices of $V(\mathcal{H})$, con-
struct the partial hypergraph $\mathcal{H}_{T}$ of $\mathcal{H}$ formed by the hy-
peredges of $\mathcal{H}$ containing at least two of the vertices of the
T. Then $\mathcal{H}$ is Helly precisely when $\mathcal{H}_{T}$ has a nonempty
core for every triple $T$.
The above algorithm corresponds to the case $p=2$
of the method for deciding if $\mathcal{H}$ is $p$-Helly. Therefore its
correctness follows from Theorem 4.2 (see Section 4$)$.
$\quad$ As for the complexity, there are $O\left(n^{3}\right)$ partial hyper-
graphs to be considered. Each one can be constructed and
checked in linear time, meaning an overall complexity of
$O\left(n^{3} \quad \sum_{E_{i} \in E(\mathcal{H})}\left|E_{i}\right|\right)=O\left(n^{4} m\right)$.

\subsection{Special hypergraphs}

We now define some classes of hypergraphs with the aim of showing that they are all Helly.

Say that $\mathcal{H}$ is an interval hypergraph when its vertices can be embedded on a line, in such a way that its hyperedges correspond to intervals of the line. An example is given in Figure $1(a)$.

A hypertree is a hypergraph $\mathcal{H}$ such that there exists a tree $T$ with vertex set $V(\mathcal{H})$ where the hyperedges of $E(\mathcal{H})$ induce subtrees in $T$. See Figure $1(b)$. Hypertrees are also called arboreal hypergraphs. The dual of hypertrees are employed in the theory of relational data bases $[45,46]$.

The following theorem characterizes hypertrees in terms of the Helly property.

Theorem 2.1 [37, 47, 81] A hypergraph $\mathcal{H}$ is a hypertree if and only if $\mathcal{H}$ is Helly and its line graph is chordal.

Next, we define more families of hypergraphs, based on the following notion. A special cycle of a hypergraph $\mathcal{H}$ is a sequence $v_{1} E_{1} v_{2} E_{2} \ldots v_{k} E_{k} v_{k+1}, k \geq 3$ and $v_{k+1}=v_{1}$, where $v_{1}, \ldots, v_{k}$ and $E_{1}, \ldots, E_{k}$ are distinct vertices and hyperedges of $\mathcal{H}$ satisfying $E_{i} \cap$ $\left\{v_{1}, \ldots, v_{k}\right\}=\left\{v_{i}, v_{i+1}\right\}$. The value $k$ is the length of the cycle.

A hypergraph is balanced if it contains no special cycle of odd length [11] and it is totally balanced if it has no special cycles of any length [67]. Finally, a hypergraph is normal if it is Helly and its line graph is perfect [67].

The following theorem asserts that all the above defined classes of hypergraphs are Helly. 


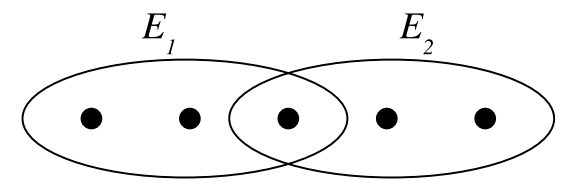

(a)

(b)

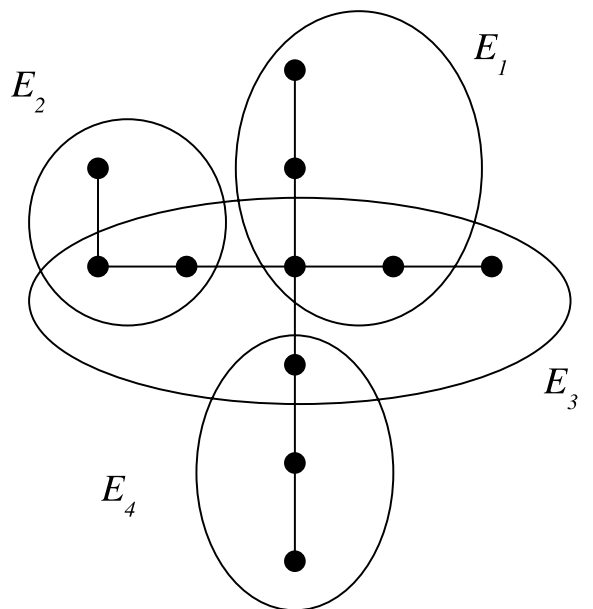

Figure 1. An interval hypergraph and a hypertree

Theorem 2.2 Normal hypergraphs, hypertrees, balanced, totally balanced, and interval hypergraphs are all Helly.

The proof of the above theorem follows from the fact that normal hypergraphs are Helly by definition, balanced hypergraphs are normal [66, 68], and totally balanced hypergraphs are balanced. On the other hand, hypertrees are Helly because the subtrees of a tree satisfy the Helly property, while interval hypergraphs are special hypertrees.

\section{Basic Helly Property on Graphs}

In the context of graphs, the Helly property has been mainly applied to certain subsets of vertices, such as cliques, disks, open neighborhoods, closed neighborhoods, and bicliques. In general, any of these special families of subsets may satisfy or not the Helly property. In this section, we consider the classes of graphs where the above families of subsets of vertices satisfy the Helly property. The clique-Helly graphs are exactly the graphs whose families of cliques satisfy the Helly property. Similarly we define disk-Helly, open neighborhood-Helly, closed neighborhood-Helly, and biclique-Helly graphs, respectively. Disk-Helly graphs are also called simply Helly graphs.

We describe characterizations and recognition algorithms for these classes, as well as show the containment relations among them. Finally, we consider another class of graphs closely related to the Helly property, the Helly circular-arc graphs.

\subsection{Clique-Helly graphs}

Clique-Helly graphs have been well studied, mainly in connection with clique graphs. The first reference to them is the following sufficient condition for a graph to be a clique graph.

Theorem 3.1 [55] Every clique-Helly graph is a clique graph.

This theorem has been generalized to an actual characterization of clique graphs, as follows:

Theorem 3.2 [80] A graph $G$ is a clique graph if and only if it contains a family of complete subsets of vertices which covers all its edges and satisfies the Helly property.

The above characterization has not lead so far to a polynomial time algorithm for recognizing clique graphs. In fact, it has been recently proved that recognizing clique graphs is NP-complete [2].

Another result closely related to Theorem 3.1 can be formulated, as follows.

Theorem 3.3 [44] The clique graph of a clique-Helly graph is clique-Helly, and every clique-Helly graph is the clique graph of a clique-Helly graph.

Clique-Helly graphs play a key role in the study of iterated clique graphs. Let $G$ and $H$ be graphs. Say that $G$ is convergent to $H$ when $K^{i}(G)=K^{i+1}(G)=H$, 
for some $i \geq 0$. When $H$ is the one-vertex graph, call $G$, simply, convergent. On the other hand, when $\lim _{i \rightarrow \infty}\left|V\left(K^{i}(G)\right)\right|=\infty, G$ is a divergent graph. Finally, when $K(G)=G$, say that $G$ is a self-clique graph.

If $G$ is clique-Helly then $K^{i}(G)$ is again cliqueHelly, and furthermore, $K^{2}(G)$ is an induced subgraph of $G$ [44]. The latter implies that divergent graphs cannot be clique-Helly. The study of divergent graphs has both algebraic and geometric connections and has attracted much interest, recently. For instance, see [59, 60, 61, 63, 75], among other papers. A general theory for this class is in $[62,72]$. Finally, as for self-clique graphs, we can mention that self-clique clique-Helly graphs have been fully characterized [15, 64]. However, little is known about self-clique graphs which are not clique-Helly. A survey on clique graphs appears in [84].

Various other classes of graphs have been defined motivated by clique-Helly graphs, or are closely related to them. See, for instance, [16, 19, 20, 85].

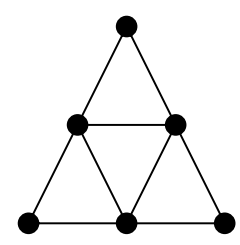

Figure 2. The Hajós Graph

The family of minimal non clique-Helly graphs has been described in [69]. Here, the minimality refers both to induced subgraphs and intersecting families of cliques.

The smallest graph which is not clique-Helly is the Hajós graph, depicted in Figure 2. In general, for recognizing clique-Helly graphs the first idea would be to apply the algorithm of Section 2.1, with the aim of checking whether the clique hypergraph of the given graph is Helly. However, since the number of cliques of a graph might be exponential [71], this would not necessarily lead to a polynomial time algorithm.

Problem 3.1 (CLIQUe-Helly GRAPH): Given a graph $G$, decide whether $G$ is clique-Helly.

However, clique-Helly graphs can be recognized in polynomial time, applying the following concept. Let $G$ be a graph and $T$ a triangle of if. The extended triangle of $G$ relative to $T$ is the subgraph induced in $G$ by the set of all vertices adjacent to at least two vertices of $T$. The following theorem characterizes clique-Helly graphs.
Theorem 3.4 [35, 83] A graph is clique-Helly if and only if each of its extended triangles contains a universal vertex.

The above theorem leads directly to a polynomial time algorithm for recognizing whether a given graph $G$ is clique-Helly.

\section{Algorithm 3.1 (RECOGNIZING}

CLIQUE-HELLY GRAPHS): For every triangle $T$ of $G$, construct its extended triangle and verify if it contains a universal vertex. Then $G$ is clique-Helly precisely when the answer is positive for every triangle $T$.

We need $O(\mathrm{~nm})$ time to generate all the triangles of $G$. The computation of the required operations, for each of the triangles, requires $O(m)$. Therefore the overall complexity is $O\left(n m^{2}\right)$. This complexity can be reduced by applying matrix multiplication for generating the triangles.

However, the following generalization of recognizing clique-Helly graphs seems to be more difficult.

A graph sandwich problem consists of given two graphs $G_{1}$ and $G_{2}$, finding a graph $G$ with some desired property, the sandwich graph, such that $E\left(G_{1}\right) \subseteq$ $E(G) \subseteq E\left(G_{2}\right)$. Graph sandwich problems were defined in the context of Computational Biology and are a natural generalization of recognition problems [50].

Problem 3.2 (CliQue-Helly SANDWICH GRAPH): Given two graphs $G_{1}, G_{2}$ such that $E\left(G_{1}\right) \subseteq E\left(G_{2}\right)$, is there a sandwich graph for $G_{1}$ and $G_{2}$ that is clique-Helly?

Theorem 3.5 [28] CliQue-Helly SANDWich GRAPH is NP-complete.

\subsection{Disk-Helly graphs}

Disk-Helly graphs can also be recognized in polynomial time. Such recognition algorithms have been described in [7, 35]. Disk-Helly graphs have been studied in connection with retracts of a graph, e.g. $[6,8,56]$. This class has been also characterized in terms of convergence, as follows.

Theorem 3.6 [8] A graph is disk-Helly if and only if it is clique-Helly and convergent.

The above theorem completely characterizes convergent graphs which are clique-Helly, implying that such a class can be recognized in polynomial time. In contrast, it is an open problem whether it is even decidable to recognize general convergent graphs. 


\subsection{Open and closed neighbourhood-Helly graphs}

Open and closed neighbourhood-Helly graphs can also be recognized in polynomial time, by applying Algorithm 2.1, since the size of the neighbourhoods is polynomially bounded. The same remark applies for disk-Helly graphs. The following definition is useful for relating clique-Helly and open neighbourhood-Helly graphs.

For a graph $G$, denote by $B(G)$ the bipartite graph with bipartition $V_{1} \cup V_{2}$, where $V_{1}=V_{2}=V(G)$, such that $v_{i} \in V_{1}$ and $v_{j} \in V_{j}$ are adjacent precisely when $v_{i}=v_{j}$ or $v_{i} v_{j} \in E(G)$.

Theorem 3.7 [6] A graph $G$ is clique-Helly if and only if $B(G)$ is open neighbourhood-Helly.

The following concept generalizes extended triangles. It has been employed both for characterizing open neighbourhood-Helly graphs and biclique-Helly graphs. For a graph $G$, let $S \subseteq V(G),|S|=3$. Denote by $B_{S}$ the family of bicliques of $G$, each of them containing at least two vertices of $S$. Let $G_{B_{S}}$ be the subgraph of $G$ formed exactly by the vertices and edges of $B_{S}$. Write $S^{*}=V\left(G_{B_{S}}\right)$. The induced subgraph of $G$ formed by the vertices of $S^{*}$ is called an extension of $S$. Finally, denote by $S_{2}^{*} \subseteq S^{*}$ the subset of vertices which are adjacent to at least two vertices of $S$.

Theorem 3.8 [53] A graph $G$ is open neighbourhoodHelly if and only if $G$ has no triangles, and for every independent set $S,|S|=3, S^{*}$ contains a vertex adjacent to all the vertices of $S_{2}^{*}$.

\subsection{Biclique-Helly graphs}

For biclique-Helly graphs, we need an additional definition. For a graph $G$, say that a vertex $v$ dominates an edge $e$ when one of the extremes of $e$ either coincides or is adjacent to $v$. When $v$ dominates every edge of $G$ then $v$ is an edge dominator of $G$. Biclique-Helly graphs can be characterized as follows.

Theorem 3.9 [53] A graph $G$ is biclique-Helly if and only if $G$ has no triangles and each of its extensions has an edge dominator.

Problem 3.3 (BIClique-Helly GRAPH): Given a graph $G$, decide whether $G$ is biclique-Helly.

As for the question of recognizing biclique-Helly graphs, first we remark that unlike neighbourhoods and disks, the number of bicliques of a graph is not polynomially bounded, meaning that a direct application of Algorithm 2.1 would not lead to an efficient method. In fact, the number of bicliques of a graph might be exponential in its number of vertices [78]. However, the above theorem can be used to formulate a polynomial time algorithm, as follows. Let $G$ be the given graph.

Algorithm 3.2 [53] (RECOGNIZING BICLIQUE-HELLY GRAPHS): First, verify if $G$ has a triangle. If it does then stop, as $G$ is not biclique-Helly. Otherwise, for each 3subset of vertices of $G$, construct its extension and verify if it contains an edge dominator. Then $G$ is biclique-Helly precisely when the answer is affirmative in all cases.

There are $O\left(n^{3}\right)$ extensions to be considered. To construct and check each of them, we require $O(m)$. The total complexity is $O\left(n^{3} m\right)$.

\subsection{Relation among classes}

Finally, we relate the Helly classes so far considered in this section. Clearly, clique-Helly graphs contain open neighborhood-Helly and biclique-Helly graphs, because the two last classes are triangle-free (Theorems 3.8 and 3.9) and every triangle-free graph is clique-Helly. Furthermore, if $T$ is a triangle of some graph $G$ whose extended triangle does not contain a universal vertex, then the closed neighbourhoods of the vertices of $T$ contain an intersecting subfamily with no common vertex. Consequently, every closed neighbourhood-Helly graph is also clique-Helly. On the other hand, it is clear that closed neighbourhood-Helly graphs contain disk-Helly graphs. However, open and closed neighbourhood-Helly graphs do not contain each other. Clearly, a triangle is closed neighbourhood-Helly and not open neighbourhood-Helly, whereas a $C_{4}$ is open neighbourhood-Helly and not closed neighbourhood-Helly. See Figure 3, where some minimal graphs are also shown.

\subsection{Helly circular-arc graphs}

A generalization of intervals of a straight line is to consider arcs of a circle, instead. However, the arcs of a circle do not form necessarily a Helly family. For example, a family of three arcs which together cover the circle, and such that none of them contains another one, is not Helly. See Figure 4. In fact, we are interested in the intersection graph of arcs of a circle, called circular-arc graph $G$. That is, $G$ has a vertex for each arc, and two vertices are adjacent when the corresponding arcs intersect. For a circular-arc graph $G$, if there exists a Helly family of arcs which represents $G$ then say that $G$ is a Helly circular-arc graph.

The above definition motivates the following problem.

Problem 3.4 (HELly CIRCULAR-ARC GRAPH): Given a graph $G$, decide whether $G$ is a Helly circular-arc graph. 


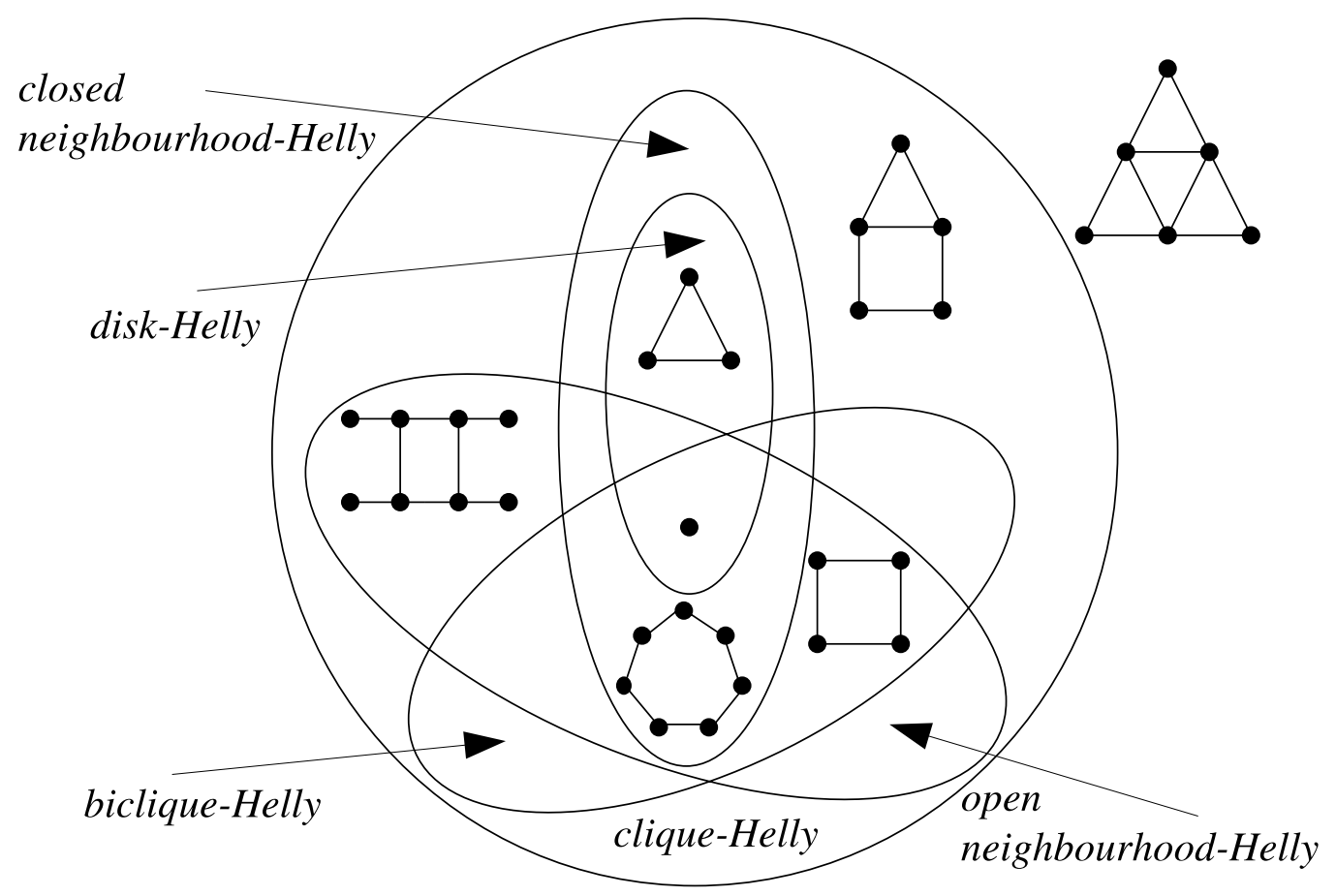

Figure 3. Relations among Helly classes
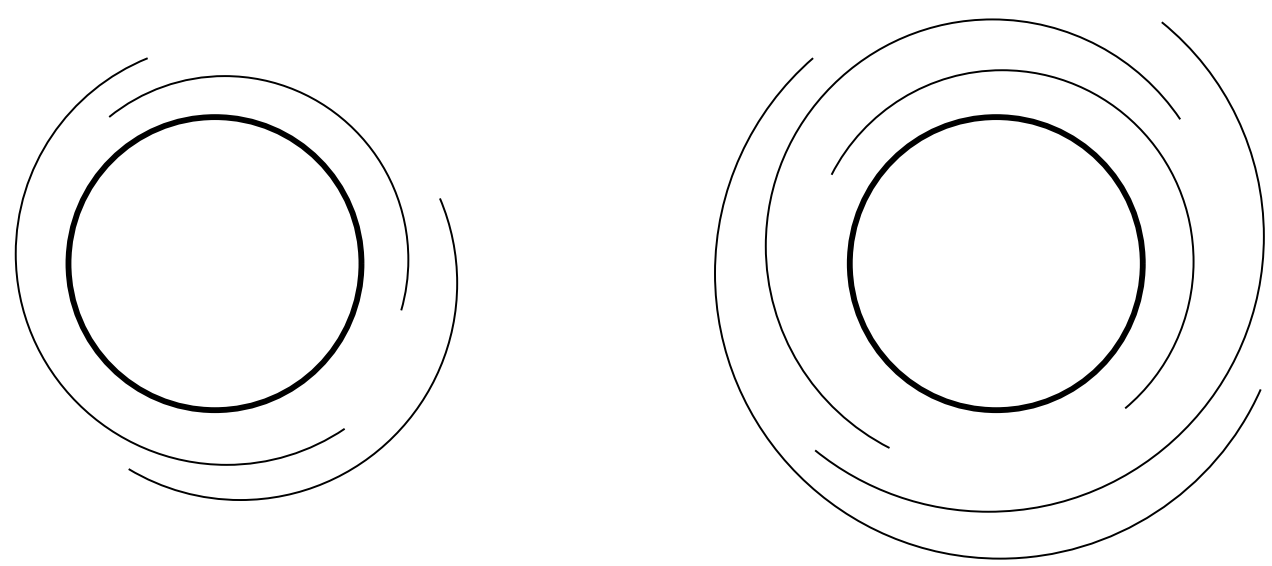

Figure 4. Non Helly families of arcs 
In order to characterize circular-arc graphs, the following concept is useful. For a $(0,1)$-matriz $M$, say that $M$ has the circular 1 's property on the columns when the 1 's in each column appear consecutively, in the ordering of the lines, considered circularly. The following theorem characterizes Helly circular-arc graphs.

Theorem 3.10 [48] A graph is a Helly circular-arc graph if and only if it admits a clique matrix possessing the circular 1 's property on the columns.

This theorem leads to the following algorithm for recognizing Helly circular-arc graphs. Let $G$ be a graph.

\section{Algorithm 3.3 [48] (RECOGNIZING HELly CIRCULAR-ARC GRAPHS): Find all cliques of $G$. If $G$ has more than $n$ cliques then stop, as $G$ is not Helly circular-arc. Otherwise, verify if its cliques can be placed in a circular ordering, so that the corresponding clique matrix has the circular 1's property on its columns. Then $G$ is a Helly circular-arc graph in the affirmative case, otherwise it is not.}

Helly circular-arc graphs can have no more than $n$ cliques, which can be computed in overall $O\left(n^{3}\right)$ time, using the algorithm in [74]. Determining whether the graph admits a clique matrix with the circular 1's property on the columns can also be done within the same bound [48]. Therefore the complexity of the algorithm is $O\left(n^{3}\right)$. See [82] for a discussion about this recognition problem.

Recently, it has been described a forbidden subgraph characterization for Helly circular-arc graphs, which leads to a linear time recognition algorithm for the class [65]. Helly circular-arc graphs have been also studied in relation to clique graphs $[16,42]$ and cliqueperfectness [17].

The corresponding recognition problem of verifying the Helly property for the case of chords of a circle, instead of arcs, has not yet been solved. A circle graph is the intersection graph of chords of a circle. A Helly circle graph is a graph admitting a representation by chords of a circle satifying the Helly property, that is, any subset of intersecting chords contains a common point. A conjecture [40] asserts that a graph is a Helly circle graph if and only if it is a circle graph with no induced subgraph isomorphic to $K_{4}-e$. See also [41].

\subsection{Matrices of a graph}

Another example of the use of the Helly property is in the characterization of clique matrices of a graph, due to Gilmore. It states that a $(0,1)$-matrix with no zero lines is the clique matrix of some graph if and only if the 1's of any row do not cover the 1's of another row, while the 1 's of the columns satisfy the Helly property. Biclique matrices of a graph have recently been characterized [54], also in terms of the Helly property.

\section{The $p$-Helly Property}

Consider the following generalization of the Helly property. A hypergraph $\mathcal{H}$ is $p$-Helly if every partial $p$ intersecting hypergraph of $\mathcal{H}$ has a nonempty core. In this section we present two characterizations of this concept, one of them leading to a polynomial-time algorithm for recognizing $p$-Helly hypergraphs when $p$ is fixed.

\section{1. $k$-Conformal hypergraphs}

Define the $k$-section of a hypergraph $\mathcal{H}$ to be a hypergraph $[\mathcal{H}]_{k}$ whose hyperedges are sets $F \subseteq V(\mathcal{H})$ such that $|F|=k$ and $F \subseteq E_{i} \in E(\mathcal{H})$; or $|F|<k$ and $F \in E(\mathcal{H})$.

For $k \geq 2$, a hypergraph $\mathcal{H}$ is $k$-conformal if every maximal set of $V(\mathcal{H})$, which induces a $K_{j}^{k}$ hypergraph of $[\mathcal{H}]_{k}$, for $k \leq j$, is a hyperedge of $\mathcal{H}$.

For example, the hypergraph $\mathcal{H}$, where $V(\mathcal{H})=$ $\{1,2,3,4\}$ and $E(\mathcal{H})=\{\{1,2\},\{1,3\},\{1,4\}\}$ is 2conformal. However, if $E(\mathcal{H})=\{\{1,2,4\},\{1,3,4\}$, $\{2,3,4\}\}$ then $\mathcal{H}$ is not 2 -conformal. There is a close relationship between $k$-conformal and $k$-Helly hypergraphs.

Theorem 4.1 [12] A hypergraph is $k$-conformal if and only if its dual is $k$-Helly.

A generalization of the above theorem, Theorem 6.6, is proved in Section 6.

\subsection{Recognition}

The following theorem characterizes $p$-Helly hypergraphs:

Theorem 4.2 [13] A hypergraph $\mathcal{H}$ is $p$-Helly if and only if for every $(p+1)$-subset $V^{\prime}$ of $V(\mathcal{H})$, the partial hypergraph formed by the hyperedges that contain at least p elements of $V^{\prime}$ has a nonempty core.

This theorem leads directly to an algorithm for recognizing if a given hypergraph $\mathcal{H}$ is $p$-Helly, for $p \geq 2$.

Problem 4.1 ( $p$-Helly HyPERGRAPH): Given a fixed integer $p$ and a hypergraph $\mathcal{H}$, decide whether $\mathcal{H}$ is $p$ Helly. 
Algorithm 4.1 [13] (RECOGNIZING $p$-HELLY HYPERGRAPHS): For each $(p+1)$-subset $V^{\prime}$ of $V(\mathcal{H})$, construct the partial hypergraph $\mathcal{H}^{\prime}$ formed by the hyperedges of $\mathcal{H}$ containing at least $p$ vertices of $V^{\prime}$, and verify if $\mathcal{H}^{\prime}$ has a nonempty core. Then $\mathcal{H}$ is $p$-Helly precisely when the answer is positive in all cases.

There are $O\left(n^{p+1}\right)$ partial hypergraphs to be considered. Each one of these partial hypergraphs as well as its core, can be constructed in $O(m(n+p))$ time. Then the overall complexity of the above algorithm is $O\left(m(n+p) n^{p+1}\right)$, that is, a polynomial for fixed $p$. As we shall see in Section 6, this problem is NP-hard for the case when $p$ is variable, since it is a particular case of Problem 6.3.

Observe that if a hypergraph is $p$-Helly, then it is $(p+1)$-Helly. From this fact, one can ask, for a given hypergraph $\mathcal{H}$, what is the least number $h$ for which $\mathcal{H}$ is $h$-Helly? This number is known as the Helly number of the hypergraph [58]. The Subsection 6.4 is dedicated to the Helly number and related topics.

What happens to the complexity of checking the $p$ Helly property if we relax the definition, in the sense that some specific partial $p$-intersecting hypergraphs with an empty core are allowed?

Let $\mathcal{H}$ be a hypergraph and $\mathscr{L}$ be a list of partial $p$ hypergraphs of $\mathcal{H}$. Say that $\mathcal{H}$ is list $p$-Helly relative to $\mathscr{L}$ if every partial $p$-intersecting hypergraph $\mathcal{H}^{\prime}$ of $\mathcal{H}$ satisfies the following condition:

- if all the partial $p$-hypergraphs of $\mathcal{H}^{\prime}$ are listed in $\mathscr{L}$, then $\operatorname{core}\left(\mathcal{H}^{\prime}\right) \neq \varnothing$.

In particular, if $\mathscr{L}$ is the list of all the partial $p$ hypergraphs of $\mathcal{H}$, then $\mathcal{H}$ is list $p$-Helly if and only if $\mathcal{H}$ is $p$-Helly.

Problem 4.2 (LIST $p$-HELly HYPERGRAPH): Let $p \geq 2$ be a fixed integer. Given a hypergraph $\mathcal{H}$ and a list $\mathscr{L}$ of partial p-hypergraphs of $\mathcal{H}$, decide whether $\mathcal{H}$ is list $p$ Helly relative to $\mathscr{L}$.

Theorem 4.3 [33] LIST $p$-HELLY HYPERGRAPH is coNP-complete.

\section{The Bounded Helly Property}

Remember that a hypergraph $\mathcal{H}$ is $p$-Helly if every $p$ intersecting partial hypergraph of $\mathcal{H}$ has a nonempty core. As an example, consider $V=\left\{a_{1}, \ldots, a_{p+1}\right\}$ and the hypergraph $\mathcal{H}$ formed by the hyperedges $V \backslash\left\{a_{i}\right\}, i=$ $1, \ldots, p+1$. Clearly, $\mathcal{H}$ is not $p$-Helly. This definition can be restricted to subfamilies of limited size.
We say that a hypergraph $\mathcal{H}$ is $k$-bounded p-Helly $(k \leq|E(\mathcal{H})|)$ if every $p$-intersecting partial $k^{-}$hypergraph of $\mathcal{H}$ has a nonempty core. This definition implies that, in a $k$-bounded $p$-Helly hypergraph, $p$ intersecting subfamilies of size strictly greater than $k$ do not necessarily contain a common element. As an example, the hypergraph defined in the previous paragraph is not $(p+1)$-bounded $p$-Helly, but it is $p$-bounded $(p-1)$ Helly. This concept, for the case $p=2$, was first considered in [80].

Observe that any hypergraph is $k$-bounded $p$-Helly for any $p \geq k$; consequently, we only need to focus the case $p<k$.

Problem 5.1 ( $k$-BOUnded $p$-Helly HYPERGRAPH): Let $p \geq 1$ and $k>p$ be fixed integers. Given a hypergraph $\mathcal{H}$, decide whether $\mathcal{H}$ is $k$-bounded $p$-Helly.

The following algorithm is straightforward from the definition.

Algorithm 5.1 (RECOGNIZING $k$-BOUNDED $p$-HELLY HYPERGRAPHS): For each partial $k^{-}$-hypergraph $\mathcal{H}^{\prime}$ of $\mathcal{H}$, verify if it is $p$-intersecting. If some $\mathcal{H}^{\prime}$ which is $p$ intersecting has an empty core, then stop, as $\mathcal{H}$ is not $k$ bounded p-Helly. Otherwise $\mathcal{H}$ is $k$-bounded p-Helly.

There are $O\left(|E(\mathcal{H})|^{k}\right)$ partial $k^{-}$-hypergraphs in $\mathcal{H}$. In order to test if one of them is $p$-intersecting and to compute its core we require $O\left(p n k^{p}\right)$ and $O(n k)$, respectively. Then the definition leads to an algorithm with time complexity $O\left(p n m^{k} k^{p}\right)$.

Problem 5.2 ( $k$-Bounded $p$-Helly HyPERGRAPH, $k$ VARIABLE): Let $p \geq 1$ be a fixed integer. Given a hypergraph $\mathcal{H}$ and an integer $k$, decide whether $\mathcal{H}$ is $k$ bounded p-Helly.

When $p$ is variable, Theorem 5.2 implies that the above problem is NP-hard. When $p$ is fixed and $k$ variable, Theorem 5.2 asserts the co-NP-completeness of Problem 5.2. The proof of it applies Theorem 5.1.

Theorem 5.1 [26] (SATISFIABILITY): Deciding whether a boolean expression in the conjuntive normal form is satisfiable is NP-complete.

Theorem 5.2 [31] $k$-BOUNDED $p$-HELLY HYPERGRAPH, $k$ VARIABLE is co-NP-complete.

Proof. It can be checked in polynomial time whether a partial $k^{-}$-hypergraph is not $p$-Helly, for fixed $p$, using Algorithm 4.1. Thus, the decision problem belongs to coNP. For the hardness proof, we employ a transformation 
from SATISFIABILITy. Let $\mathcal{E}$ be a boolean expression. Denote by $X=\left\{x_{1}, \ldots, x_{n}\right\}$ the set of variables of $\mathcal{E}$ and by $\mathscr{C}=\left\{C_{1}, \ldots, C_{m}\right\}$ the set of clauses. Build a hypergraph $\mathcal{H}$ in the following way: for each variable of $X$ and each clause of $\mathscr{C}$ create one vertex in $\mathcal{H}$. Denote $V(\mathcal{H})=V_{X} \cup V_{\mathscr{C}}$, where $V_{X}=\left\{v_{1}, \ldots, v_{n}\right\}$ and $V_{\mathscr{C}}=\left\{c_{1}, \ldots, c_{m}\right\}$, that is, the vertex $v_{i} \in V_{X}$ is associated to the variable $x_{i} \in X$ and the vertex $c_{j} \in V_{\mathscr{C}}$ to the clause $C_{j} \in \mathscr{C}$. For each variable $x_{i} \in X$ create the hyperedges $E_{x_{i}}$ and $E_{\bar{x}_{i}}$ in $\mathcal{H}$, adding to them the vertices of $V_{X} \backslash\left\{v_{i}\right\}$. Furthermore, for each vertex $c_{j} \in V_{\mathscr{C}}$, include $c_{j}$ in the hyperedge $E_{x_{i}}\left(E_{\bar{x}_{i}}\right)$ if and only if the literal $x_{i}\left(\bar{x}_{i}\right)$ does not appear in the clause $C_{j}$. Finally, define $k=n$.

Let $\mathcal{H}^{\prime}$ be a partial hypergraph of $\mathcal{H}$. If $\mathcal{H}^{\prime}$ does not contain at least one of the hyperedges $E_{x_{i}}$ or $E_{\bar{x}_{i}}$, corresponding to $x_{i} \in X$, then $v_{i}$ belongs to the core of $\mathcal{H}^{\prime}$. Hence, in order to verify whether $\mathcal{H}$ is $k$-bounded $p$-Helly we need to consider only the partial hypergraphs $\mathcal{H}^{\prime}$ with exactly $k$ hyperedges such that, for every variable $x_{i} \in X$, either $E_{x_{i}}$ or $E_{\bar{x}_{i}}$ is a hyperedge of $\mathcal{H}^{\prime}$. Then let $\mathcal{H}^{\prime}$ be a partial $k$-hypergraph of $\mathcal{H}$ satisfying such a property. Clearly, every $v_{i} \in V_{X}$ does not belong to the core of $\mathcal{H}^{\prime}$ and $\mathcal{H}^{\prime}$ is $(k-1)$-intersecting. Hence, $\mathcal{H}^{\prime}$ is $p$-intersecting, because $p<k$.

Since $\mathcal{H}^{\prime}$ contains $E_{x_{i}}$ or $E_{\bar{x}_{i}}$ for every $x_{i} \in X, \mathcal{H}^{\prime}$ defines a truth assignment for $\mathscr{C}$. In this truth assignment a literal has the value true if and only if the corresponding hyperedge belongs to $\mathcal{H}^{\prime}$. Therefore, let us say that $\mathcal{H}^{\prime}$ satisfies $\mathcal{E}$ if and only if this truth assignment satisfies $\mathcal{E}$.

Suppose that $\mathcal{H}^{\prime}$ satisfies $\mathcal{E}$. Then any clause of $\mathscr{C}$ contains at least one literal associated to some hyperedge of $\mathcal{H}^{\prime}$. This means that for each vertex $c_{i} \in V_{\mathscr{C}}$ there exists one hyperedge in $\mathcal{H}^{\prime}$ which does not contain it; therefore the core of $\mathcal{H}^{\prime}$ is empty, meaning that $\mathcal{H}$ is not $k$ bounded $p$-Helly.

Conversely, suppose that $\mathcal{H}^{\prime}$ does not satisfy $\mathcal{E}$, and let $C_{j} \in \mathscr{C}$ be a clause in which no literal has the value true. Consider an arbitrary variable $x_{i} \in X$. If $E_{x_{i}}$ is the edge of $\mathcal{H}^{\prime}$ representing $x_{i}$ then $x_{i} \notin C_{j}$, otherwise $C_{j}$ would be satisfied. This implies $c_{j} \in E_{x_{i}}$. Similarly, whenever $E_{\bar{x}_{i}}$ is the representing edge of $x_{i}$, we have $c_{j} \in E_{\bar{x}_{i}}$. In either case, $c_{j}$ belongs to the edge representing $x_{i}$, for every $i$. Thus $c_{j}$ belongs to the core of $\mathcal{H}^{\prime}$, that is, $\mathcal{H}$ is $k$-bounded $p$-Helly.

Applying this concept to the cliques of a graph, we have the $k$-bounded $p$-clique-Helly graphs. Consider now the recognition problem for graphs. The next theorem states that this problem is co-NP-complete, even for fixed $k$ and $p$.
Problem 5.3 ( $k$-BOUNDED $p$-CLIQUE-HELlY GRAPH): Let $k>p \geq 1$ be fixed integers. Given a graph $G$, decide whether $G$ is a k-bounded p-clique-Helly graph.

\section{Theorem 5.3 [31] $k$-BOUnded $p$-CLIQUE-Helly} GRAPH is co-NP-complete.

By the definition, it is clear that Clique-Helly $\subset$ $k$-BOUNDED CLIQUE-HELlY $\subset k^{\prime}$-BOUNDED CLIQUEHELLY, for $k^{\prime}<k$.

However, for $K_{k+1}$-free graphs, the classes of cliqueHelly and $k$-bounded clique-Helly coincide.

Lemma 5.1 [80] $A K_{k+1}$-free graph is clique-Helly if and only if it is $k$-bounded clique-Helly.

Let $G$ be a planar graph. Since any planar graph is $K_{5}$-free, the number of cliques of $G$ is $O\left(n^{4}\right)$. Using Algorithm 5.1, we conclude that the next problem can be solved in polynomial time.

Problem 5.4 (Planar 3-BOUNDED CLIQUe-Helly GRAPH): Given a graph $G$, decide whether $G$ is planar $k$-bounded clique-Helly.

A characterization which leads to a good algorithm for recognizing planar 3-bounded clique-Helly graphs is presented in [3], Next, we describe it.

For a given triangle $T=\{x, y, z\}$ of $G$, we call:

- $V_{x y}=\{v \in V(G): v \in N[x], v \in N[y], v \notin$ $N[z]\}$;

- $V_{x z}=\{v \in V(G): v \in N[x], v \in N[z], v \notin$ $N[y]\}$

- $V_{y z}=\{v \in V(G): v \in N[y], v \in N[z], v \notin$ $N[x]\}$;

- $V_{x y z}=\{v \in V(G): v \in N[x], v \in N[y]$, $v \in N[z]\}$.

Let $G$ be a graph and $T^{\prime}$ the extended triangle of $G$ relative to the triangle $T=\{x, y, z\}$. Say that:

- $T^{\prime}$ is of type 1 if at least one of the sets $V_{x y}, V_{x z}$ or $V_{y z}$ is empty;

- $T^{\prime}$ is of type 2 if $V_{x y}=\left\{z_{1}\right\}, V_{x z}=\left\{y_{1}\right\}$, $V_{y z}=\left\{x_{1}\right\}, V_{x y z}=\{w\}$, and $w$ is adjacent to $x_{1}$, $y_{1}$ and $z_{1}$;

- $T^{\prime}$ is of type 3 if $V_{x y}=\left\{z_{1}\right\}, V_{x z}=\left\{y_{1}\right\}, V_{y z}=$ $\left\{x_{1}\right\}, V_{x y z}=\left\{w, w^{\prime}\right\}$, and $w$ is adjacent to $x_{1}, y_{1}$ and $z_{1}$. 


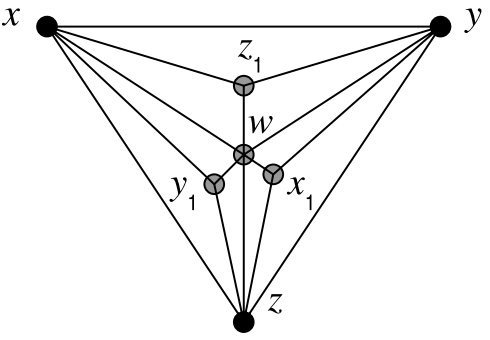

(a)

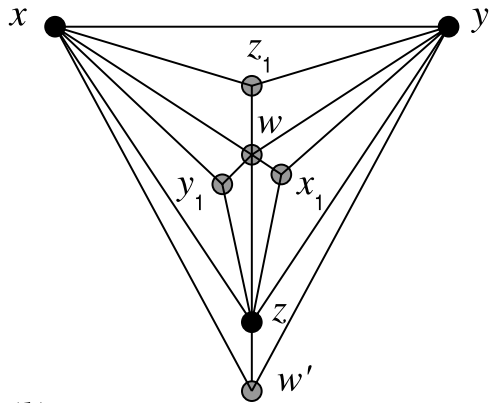

(b)

Figure 5. Extended triangles of type 2 and 3

Notice that if $T^{\prime}$ is an extended triangle of type 2, or type 3, of a planar graph, then $T^{\prime}$ is isomorphic to the graph $(a)$, or $(b)$, in Figure 5, respectively.

Theorem 5.4 [3] Let $G$ be a planar graph.

1. $G$ is a clique-Helly graph if and only if every extended triangle of $G$ is of type 1 or type 2 .

2. G is a 3-bounded clique-Helly graph if and only if every extended triangle of $G$ is of type 1 , type 2 or type 3.

By this characterization, assintotically, the complexities to recognize clique-Helly planar graphs and 3bounded clique-Helly planar graphs are the same. Therefore we discuss only the algorithm for 3-bounded 2clique-Helly planar graphs.

Algorithm 5.2 (RECOGNIZING 3-BOUNDED CLIQUEHELLY GRAPHS): Construct the extended triangle for every triangle of $G$. If some extended triangle is not of type 1, type 2, nor of type 3 , then stop as the graph is not 3-bounded clique-Helly graph, otherwise it is.

Since the triangles of a planar graph can be listed in $O(n)$ time [73], the above algorithm has complexity $O\left(n^{2}\right)$.

\section{Cardinality of the Intersections on Hyper- graphs}

In this section we extend the idea of the Helly property by considering the cardinality of the intersections. Such concept was introduced in [91].

\section{1. $(p, q)$-Intersecting}

We begin with a generalization of $p$-intersecting. Let $p \geq 1$ and $q \geq 0$. A hypergraph $\mathcal{H}$ is $(p, q)$-intersecting when every partial $p^{-}$-hypergraph of $\mathcal{H}$ has a $q^{+}$-core.

Clearly, the following implications hold for any hypergraph $\mathcal{H}$.

- If $1 \leq q \leq|\operatorname{core}(\mathcal{H})|$, then $\mathcal{H}$ is $(p, q)$-intersecting.

- For $p \geq 2$, if $\mathcal{H}$ is $(p, q)$-intersecting, then $\mathcal{H}$ is $(p-$ $1, q)$-intersecting.

- If $\mathcal{H}$ is $(p, q)$-intersecting, then $\mathcal{H}$ is $(p, q-1)$ intersecting.

- $\mathcal{H}$ is $(1, q)$-intersecting if and only if every hyperedge of $\mathcal{H}$ contains at least $q$ vertices.

- If $\mathcal{H}$ is $(p, q)$-intersecting, then every partial hypergraph of $\mathcal{H}$ is $(p, q)$-intersecting.

If $p$ is fixed, then it is possible to test whether $\mathcal{H}$ is $(p, q)$-intersecting in polynomial time by simply computing the core of every partial $p$-hypergraph of $\mathcal{H}$. For the case in which $p$ is not fixed it was proved that deciding whether $\mathcal{H}$ is $(p, q)$-intersecting is co-NP-complete.

Problem 6.1 $((p, q)$-InTERSECTING HyPERGRAPH, $p$ VARIABLE): Let $q$ be a fixed positive integer. Given $p \geq 1$ and a hypergraph $\mathcal{H}$, decide whether $\mathcal{H}$ is $(p, q)$ intersecting.

Theorem 6.1 [33] $(p, q)$-INTERSECTING HYPERGRAPH, $p$ VARIABLE is co-NP-complete. 


\section{2. $(p, q, s)$-Helly hypergraphs}

The following definition is a generalization of the $p$ Helly property, and has been introduced in [91].

Let $p \geq 1, q \geq 0$ and $s \geq 0$. A hypergraph $\mathcal{H}$ is $(p, q, s)$-Helly when every partial $(p, q)$-intersecting hypergraph of $\mathcal{H}$ has an $s^{+}$-core.

The following implications are true for any hypergraph $\mathcal{H}$.

- If $\mathcal{H}$ is $(p, q, s)$-Helly, then $\mathcal{H}$ is $(p+1, q, s)$-Helly.

- If $\mathcal{H}$ is $(p, q, s)$-Helly, then $\mathcal{H}$ is $(p, q+1, s)$-Helly.

- If $\mathcal{H}$ is $(p, q, s)$-Helly, then $\mathcal{H}$ is $(p, q, s-1)$-Helly.

- $\mathcal{H}$ is $(1, q, s)$-Helly if and only if the partial hypergraph formed by the $q^{+}$-hiperedges of $\mathcal{H}$ has an $s^{+}$core.

The following definitions are employed in a characterization of $(p, q, s)$-Helly hypergraphs. Let $\mathcal{H}_{1}$ and $\mathcal{H}_{2}$ be hypergraphs. Then $\mathcal{H}_{1} \triangleright \mathcal{H}_{2}$ is the partial hypergraph of $\mathcal{H}_{1}$ defined in the following way:

$$
\begin{gathered}
\mathcal{H}_{1} \triangleright \mathcal{H}_{2}=\left\{E \in E\left(\mathcal{H}_{1}\right): E\right. \text { contains at least } \\
\left.\left|E\left(\mathcal{H}_{2}\right)\right|-1 \text { hyperedges of } \mathcal{H}_{2}\right\} .
\end{gathered}
$$

Lemma 6.1 Let $\mathcal{H}_{1}$ and $\mathcal{H}_{2}$ be hypergraphs and $t=$ $\left|E\left(\mathcal{H}_{2}\right)\right| \geq 2$. If $\mathcal{H}_{1}^{\prime}$ is a partial hypergraph of $\mathcal{H}_{1} \triangleright \mathcal{H}_{2}$ and $0<\left|E\left(\mathcal{H}_{1}^{\prime}\right)\right|<t$, then core $\left(\mathcal{H}_{1}^{\prime}\right)$ contains at least $t-\left|E\left(\mathcal{H}_{1}^{\prime}\right)\right|$ hyperedges of $\mathcal{H}_{2}$.

Theorem 6.2 [32] Let $p \geq 2, q \geq 1, s \geq 1$ and $\mathcal{H} a$ hypergraph. Define $a=\max \{q-s, 0\}, b=\min \{q, s\}$ and $\mathcal{I}$ as the $K_{n}^{b}$ hypergraph with vertex set $V(\mathcal{H})$. Then $\mathcal{H}$ is $(p, q, s)$-Helly if and only if:

(i) for every partial $(p+a+1)$-hypergraph $\mathcal{H}^{\prime}$ of $\mathcal{I}$, if the hypergraph $\mathcal{H} \triangleright \mathcal{H}^{\prime}$ is $(p, q)$-intersecting then it has an $s^{+}$-core; and

(ii) every partial $(p, q)$-intersecting $(p+a)^{-}$hypergraph of $\mathcal{H}$ has an $s^{+}$-core.

Proof. If $\mathcal{H}$ is $(p, q, s)$-Helly then Conditions (i) and (ii) are clearly satisfied by the definition. Assume now that $\mathcal{H}$ is not $(p, q, s)$-Helly. Then there exists a partial $(p, q)$ intersecting hypergraph $\mathcal{H}^{\prime}$ of $\mathcal{H}$ such that $\left|\operatorname{core}\left(\mathcal{H}^{\prime}\right)\right|<$ $s$.

If $\left|E\left(\mathcal{H}^{\prime}\right)\right| \leq p+a$ then $\mathcal{H}^{\prime}$ is a partial $(p, q)$ intersecting $(p+a)^{-}$-hypergraph of $\mathcal{H}$ that violates Condition (ii).

Otherwise, if $\left|E\left(\mathcal{H}^{\prime}\right)\right| \geq p+a+1$, write $E\left(\mathcal{H}^{\prime}\right)=$ $\left\{E_{1}, \ldots, E_{\left|E\left(\mathcal{H}^{\prime}\right)\right|}\right\}$ and assume that $\mathcal{H}^{\prime}$ is minimal, that is, $E\left(\mathcal{H}^{\prime}\right)-E^{\prime}$ has an $s^{+}$-core, for any $E^{\prime} \in E\left(\mathcal{H}^{\prime}\right)$. (If $\mathcal{H}^{\prime}$ is not minimal, one can successively remove hyperedges from $\mathcal{H}^{\prime}$ until obtaining either a minimal $(p+$ $a+1)^{+}$-hypergraph or a $(p+a)^{-}$-hypergraph violating Condition (ii)).

For each $i, 1 \leq i \leq\left|E\left(\mathcal{H}^{\prime}\right)\right|$, let $S_{i} \subseteq \operatorname{core}\left(\mathcal{H}^{\prime}-E_{i}\right)$ be a $b$-subset of vertices such that $S_{i} \nsubseteq E_{i}$ and $S_{i} \subseteq E_{j}$ for every $j \neq i$. This means that there exists $v_{i} \in S_{i}$ such that $v_{i} \notin E_{i}$ but $v_{i} \in E_{j}$ for every $j \neq i$.

Let $\mathcal{H}_{1}$ be the hypergraph formed by the hyperedges $S_{1}, \ldots, S_{p+a+1}$. Note that $\mathcal{H}_{1}$ is a partial $(p+a+1)$ hypergraph of $\mathcal{I}$. Define $\mathcal{H}^{\prime \prime}=\mathcal{H} \triangleright \mathcal{H}_{1}$. Since $E\left(\mathcal{H}^{\prime}\right) \subseteq$ $E\left(\mathcal{H}^{\prime \prime}\right), \mathcal{H}^{\prime \prime}$ does not have an $s^{+}$-core. Let us show that $\mathcal{H}^{\prime \prime}$ is $(p, q)$-intersecting.

Consider any partial $p$-hypergraph $\mathcal{H}^{\prime \prime \prime}$ of $\mathcal{H}^{\prime \prime}$. By Lemma 6.1, $\operatorname{core}\left(\mathcal{H}^{\prime \prime \prime}\right)$ contains at least $a+1$ hyperedges of $\mathcal{H}_{1}$, say $S_{1}, \ldots, S_{a+1}$. Note that $S_{1} \cup\left\{v_{i}: 2 \leq i \leq\right.$ $a+1\}$ contains exactly $b+a=q$ vertices. This means that $\left|\operatorname{core}\left(\mathcal{H}^{\prime \prime \prime}\right)\right| \geq q$. Therefore, $\mathcal{H}^{\prime \prime}$ is $(p, q)$-intersecting and does not have an $s^{+}$-core. This violates Condition (i).

Problem 6.2 $((p, q, s)$-Helly HYPERGRAPH, $s$ VARI$\mathrm{ABLE})$ : Let $p, q \geq 1$ be fixed integers. Given a hypergraph $\mathcal{H}$ and $s \geq 1$, decide whether $\mathcal{H}$ is $(p, q, s)$-Helly.

The above theorem leads to the following algorithm for Problem 6.2. Let $\mathcal{H}$ be a hypergraph and $\mathcal{I}$ be the hypergraph $K_{n}^{b}$ with vertex set $V(\mathcal{H})$.

Algorithm 6.1 (RECOGNIZING $(p, q, s)$-HELLY HYPERGRAPHS):

Part (i): for each partial $(p+a+1)$-hypergraph $\mathcal{H}^{\prime}$ of $K_{\mathcal{H}}^{b}$, construct the partial hypergraph $\mathcal{H}^{\prime \prime}$ choosing the hyperedges containing at least $p+a$ hyperedges of $\mathcal{H}^{\prime}$. Then verify if $\mathcal{H}^{\prime \prime}$ is $(p, q)$-intersecting. If so, verify if $\mathcal{H}^{\prime \prime}$ has an $s^{+}$-core.

Part (ii): for each partial $(p+a)^{-}$-hypergraph of $\mathcal{H}$, check if it is $(p, q)$-intersecting. If so, verify if it has an $s^{+}$-core.

The complexity of Part $(i)$ of the above algorithm is $O\left(p m^{p} n^{b(p+a+1)+1}\right)$, because there are $O\left(n^{b(p+a+1)}\right)$ partial $(p+a+1)$-hypergraphs in $\mathcal{I}$, and for each one we spend $(m(n+(p+a) b))$ steps to construct every $\mathcal{H}^{\prime \prime}$, $O\left(p^{p} m^{p}\right)$ to check if it is $(p, q)$-intersecting and $O(n m)$ to compute its core. For Part $(i i)$, the complexity is $O\left(p n m^{p+a}(p+a)^{p+1}\right)$. The overall time complexity is the sum of both. This means that it is polynomial, for $p, q$ fixed and $s$ variable. 
Problem 6.3 $((p, q, s)$-HeLly HYPERGRAPH, $p$ VARIABLE): Let $q, s \geq 1$ be fixed integers. Given a hypergraph $\mathcal{H}$ and $p \geq 2$, decide whether $\mathcal{H}$ is $(p, q, s)$-Helly.

Theorem 6.3 [33] $(p, q, s)$-HELLY HYPERGRAPH, $p$ VARIABLE is NP-hard.

We deal now with the case in which $q$ is not fixed.

Problem 6.4 $((p, q, s)$-Helly HYPERGRAPH, $q$ VARIABLE): Let $p \geq 2$ and $s \geq 1$ be fixed integers. Given a hypergraph $\mathcal{H}$ and $q \geq 1$, decide whether $\mathcal{H}$ is $(p, q, s)$ Helly.

Theorem 6.4 [33] $(p, q, s)$-HELly HYPERGRAPH, $q$ VARIABLE is co-NP-complete.

\subsection{The case $q=s$}

The case $q=s$ is natural and interesting. For simplicity, we write $(p, q)$-Helly hypergraphs, meaning $(p, q, q)$ Helly hypergraphs. In special, bounds for $(2, q)$-Helly hypergraphs were described in [90].

The following problem was proposed in [90].

Problem $6.5((p, q)$-Helly HYPERGRAPH, $q$ VARIABLE): Find a structural characterization of $r$-uniform $(2, q)$-Helly hypergraphs for $r>q+1$.

This problem remains open. However, if we consider $q=s$ fixed, we have a polynomial algorithm as a consequence of Theorem 6.2. In this case, Condition (ii) of Theorem 6.2 is trivially satisfied. Then we can rewrite the characterization as follows.

Corollary 6.1 [32] A hypergraph $\mathcal{H}$ is $(p, q)$-Helly if and only if $\mathcal{H} \triangleright \mathcal{H}^{\prime}$ has a $q^{+}$-core for every partial $(p+1)$-hypergraph $\mathcal{H}^{\prime}$ of the hypergraph $K_{n}^{q}$ with vertex set $V(\mathcal{H})$.

Problem 6.6 $((p, q)$-HELly HYPERGRAPH, $q$ FIXED): Let $q$ be a fixed integer. Given a hypergraph $\mathcal{H}$, decide whether $\mathcal{H}$ is $(p, q)$-Helly.

The complexity of recognizing $(p, q)$-Helly hypergraphs, given by this characterization and using Algorithm 6.1 , is $O\left(n^{q(p+1)} m(n+p q)\right)$. For the case $p=2$, we have $O\left(m n^{3 q+1}\right)$. Note also that, if $q=1$, we obtain the same complexity as that of Algorithm 4.1.

Now we present two attempts to solve Problem 6.5, which provide a solution for the case $q$ variable with the restriction that $r-q$ is small.

Problem 6.7 $((2, q)$-Helly HYPERGRAPH, $r-q$ FIXED): Let $q$ be an integer and $\mathcal{H}$ a hypergraph with rank $r$. Decide whether $\mathcal{H}$ is $(2, q)$-Helly.
The first one is a consequence of the following proposition.

Proposition 6.1 [90] If $\mathcal{H}$ is a minimal non- $(2, q)$-Helly hypergraph of rank $r$ with $1 \leq q<r$, then $|E(\mathcal{H})| \leq$ $r-q+2$.

Algorithm 6.2 $((2, q)$-HELly HYPERGRAPHS ): For every partial $(r-q+2)^{-}$-hypergraph of a hypergraph $\mathcal{H}$, compute its core and verify if it contains at least $q$ elements. Then $\mathcal{H}$ is $(2, q)$-Helly precisely when the answer is affirmative in all cases.

There are $\sum_{i=3}^{r-q+2}\left(\begin{array}{c}m \\ i\end{array}\right)=O\left(m^{r-q+3}\right)$ partial hypergraphs to be considered. In order to compute the core of each one we need $O(\mathrm{~nm})$ time. The overall complexity is $O\left(n m^{r-q+4}\right)$.

We present in the sequel another way to verify if a hypergraph is $(2, q)$-Helly. The $q$-line graph of a hypergraph $\mathcal{H}$, denoted by $L_{q}(\mathcal{H})$, has a vertex for every hyperedge of $\mathcal{H}$ and two vertices are adjacent if the corresponding hyperedges share at least $q$ vertices.

Theorem 6.5 The number of cliques of $L_{q}(\mathcal{H})$ for a $(2, q)$-Helly hypergraph $\mathcal{H}$ of rank $r$ is not greater than $m\left(\begin{array}{l}r \\ q\end{array}\right)$.

Proof. Let $\mathcal{H}$ be a $(2, q)$-Helly hypergraph. First note that if $C^{\prime}, C^{\prime \prime}$ are cliques of $L_{q}(\mathcal{H})$ and $\mathcal{H}^{\prime}, \mathcal{H}^{\prime \prime}$ are the partial hypergraphs of $\mathcal{H}$ formed by the hyperedges associated to the vertices of $C^{\prime}$ and $C^{\prime \prime}$, respectively, then the cores of $\mathcal{H}^{\prime}$ and $\mathcal{H}^{\prime \prime}$ are incomparable and each one has at least $q$ elements.

Let $v$ be a vertex of $L_{q}(\mathcal{H})$ and $E_{v}$ be the hyperedge of $\mathcal{H}$ corresponding to $v$. Since $E_{v}$ contains all the cores of the partial hypergraphs associated to the cliques which $v$ belongs to, $v$ appears in at most $\left(\begin{array}{l}r \\ q\end{array}\right)$ cliques of $L_{q}(\mathcal{H})$. Since the number of vertices of $L_{q}(\mathcal{H})$ is $m$, the number of cliques of $L_{q}(\mathcal{H})$ is not greater than $m\left(\begin{array}{l}r \\ q\end{array}\right)$.

The above theorem leads to the following algorithm. Let $\mathcal{H}$ be a hypergraph, and $q \geq 0$ an integer.

Algorithm 6.3 ((2, q)-HELLY HYPERGRAPHS):

Construct the graph $L_{q}(\mathcal{H})$, and generate its cliques, $C_{1}, \ldots, C_{i}, \ldots$ For each $C_{i}$, proceed as follows.

- If $i>\frac{m r !}{q !(r-q) !}$ then stop: $\mathcal{H}$ is not $(2, q)$-Helly.

- Otherwise, construct the partial hypergraph $\mathcal{H}_{i}$ of $\mathcal{H}$, formed by the hyperedges of $\mathcal{H}$ corresponding to the vertices of $C_{i}$. If core $\left(\mathcal{H}_{i}\right)<q$, then stop: $\mathcal{H}$ is not $(2, q)$-Helly. 
Otherwise, if all the cliques of $L_{q}(\mathcal{H})$ have been generated, then stop: $\mathcal{H}$ is $(2, q)$-Helly.

To construct $L_{q}(\mathcal{H})$ we spend $O\left(m^{2} n\right)$ steps. We generate at most $m\left(\begin{array}{l}r \\ q\end{array}\right)$ cliques, each with time complexity $n m$ by the algorithm of [86]. To compute the core of each partial hypergraph, $O(\mathrm{~nm})$ steps are required. The total complexity of the above algorithm is $O\left(m^{2} n+n^{2} m^{3}\left(\begin{array}{l}r \\ q\end{array}\right)\right)=O\left(n^{2} m^{3}\left(\begin{array}{l}r \\ q\end{array}\right)\right)$.

The time complexity of this algorithm for solving Problem 2, when $q$ is fixed, is $O\left(n^{2} m^{3} r^{q}\right)=$ $O\left(n^{q+2} m^{3}\right)$, while for Question 3, when $r-q$ is small, we require $O\left(n^{2} m^{3} r^{r-q}\right)=O\left(n^{r-q+2} m^{3}\right)$ steps.

\subsection{Helly numbers}

A hypergraph $\mathcal{H}$ has Helly number $h$ if $h$ is the least number for which $\mathcal{H}$ is $h$-Helly [58]. For the general $(p, q, s)$-Helly property it is possible to define variations of the Helly number in the following ways:

- Let $q, s \geq 0$ be fixed integers. The $\left(p^{*}, q, s\right)$-Helly number of $\mathcal{H}$ is the least integer $p$, if it exists, such that $\mathcal{H}$ is $(p, q, s)$-Helly.

- Let $p \geq 1$ and $s \geq 0$ be fixed integers. The $\left(p, q^{*}, s\right)$-Helly number of $\mathcal{H}$ is the least integer $q$ such that $\mathcal{H}$ is $(p, q, s)$-Helly. This number is well defined since $\mathcal{H}$ is $(p, n+1, s)$-Helly for any $p, s$.

- Let $p \geq 1$ and $0 \leq q \leq r(\mathcal{H})$ be fixed integers. The $\left(p, q, s^{*}\right)$-Helly number of $\mathcal{H}$ is the largest $s$ for which $\mathcal{H}$ is $(p, q, s)$-Helly.

By Theorem 6.3, we conclude that determining the $\left(p^{*}, q, s\right)$-Helly number is NP-hard. Similarly, Theorem 6.4 implies that finding the $\left(p, q^{*}, s\right)$-Helly number is also NP-hard. However, using Theorem 6.1, we can determine the $\left(p, q, s^{*}\right)$-Helly number in polynomial time.

\section{5. $(p, q)$-Conformal hypergraphs}

Now we generalize Theorem 4.1. In order to do so, we use the following generalizations of the concepts of $k$-section and $k$-conformal hypergraphs.

Define the $(p, q)$-section of $\mathcal{H}$ to be a hypergraph $[\mathcal{H}]_{p, q}$ whose hyperedges are sets $F \subseteq V(\mathcal{H})$ such that $|F|=p$ and $F$ is contained in at least $q$ hyperedges of $\mathcal{H}$; or $|F|<p$ and $F$ is a maximal set contained in at least $q$ hyperedges of $\mathcal{H}$.

A hypergraph $\mathcal{H}$ is $(p, q)$-conformal if every maximal set of $V(\mathcal{H})$, which induces a $K_{j}^{p}$ hypergraph of $[\mathcal{H}]_{p, q}$, for $p \leq j$, is contained in at least $q$ hyperedges of $\mathcal{H}$.

Theorem 6.6 A hypergraph $\mathcal{H}$ is $(p, q)$-Helly if and only if its dual is $(p, q)$-conformal.

Proof. Let $\mathcal{H}$ be a hypergraph and $\mathcal{H}^{*}$ its dual hypergraph. Suppose first that $\mathcal{H}^{*}$ is not $(p, q)$-conformal.
Hence in $\left[\mathcal{H}^{*}\right]_{p, q}$ there is a maximal set that induces a $p$ complete hypergraph $\mathcal{I}$, such that $V(\mathcal{I})$ is not contained in $q$ hyperedges of $\mathcal{H}^{*}$. However, the hyperedges of $\mathcal{H}$, associated to the vertices of $\mathcal{I}$, form a $(p, q)$-intersecting partial hypergraph with no $q^{+}$-core.

Conversely, suppose that $\mathcal{H}$ is not $(p, q)$-Helly. Consider a maximal $(p, q)$-intersecting partial hypergraph $\mathcal{H}^{\prime}$ of $\mathcal{H}$ with no $q^{+}$-core. The hyperedges of $\mathcal{H}^{\prime}$ correspond to a subset of vertices $C$ of $\mathcal{H}^{*}$ with the property that every $p$ of them belong to at least $q$ hyperedges of $\mathcal{H}^{*}$ simultaneously. This means that $C$ is a maximal set inducing a $p$-complete partial hypergraph $\mathcal{I}$ of $\left[\mathcal{H}^{*}\right]_{p, q}$. Furthermore, if $V(\mathcal{I})=C$ is contained in at least $q$ hyperedges of $\mathcal{H}^{*}$, this implies that $\mathcal{H}^{\prime}$ contains a $q^{+}$-core, which contradicts the hypothesis.

\section{Cardinality of the Intersections on Cliques of Graphs}

In this section we apply the $(p, q, s)$-Helly property to the clique hypergraph of a graph. Thus, a graph is $(p, q, s)$-clique-Helly if its clique hypergraph is $(p, q, s)$ Helly. According to this definition, (2,1)-clique-Helly graphs are the clique-Helly graphs. First we focus on the recognition problem of the case $q=s$, which we call $(p, q)$-clique-Helly graphs, and after we deal with the problem of deciding if the clique graph of a graph is clique-Helly.

\section{1. $(p, q)$-Clique-Helly graphs}

We begin with an example. Define, for two integers $p$ and $q$, the graph $G_{p, q}$ as follows: $V\left(G_{p, q}\right)$ is formed by a $(q-1)$-complete set $Q$, a $p$-complete set $Z=\left\{z_{1}, \ldots, z_{p}\right\}$, and a $p$-independent set $W=$ $\left\{w_{1}, \ldots, w_{p}\right\}$. Further, there are the edges $z_{i} w_{j}$, for $i \neq j$, and the edges $q x$, for $q \in Q$ and $x \in Z \cup W$.

The general graph $G_{p, q}$ appears in Figure 6, where a thick line joining two sets means that every vertex of a set is adjacent to all vertices of the other. Furthermore, for every vertex of $Z$, there is a dotted line joining it to the only vertex of $W$ which is not adjacent to it.

The graph $G_{p, q}$ contains exactly $p+1$ cliques of size $p+q-1$, namely: $Q \cup Z$ and $Q \cup\left(Z \backslash\left\{z_{i}\right\}\right) \cup\left\{w_{i}\right\}$, for $1 \leq i \leq p$.

Observe that $G_{p, q}$ is $(p, q)$-clique-Helly, but it is not $(p-1, q)$-clique-Helly. Therefore, $G_{p, q}$ is $(t, q)$-cliqueHelly for $t \geq p$, and is not $(t, q)$-clique-Helly for $t<p$.

Furthermore, $G_{p+1, q}$ is not $(p, q)$-clique-Helly, but it is $(p, t)$-clique-Helly for any $t \neq q$. Consequently, for 


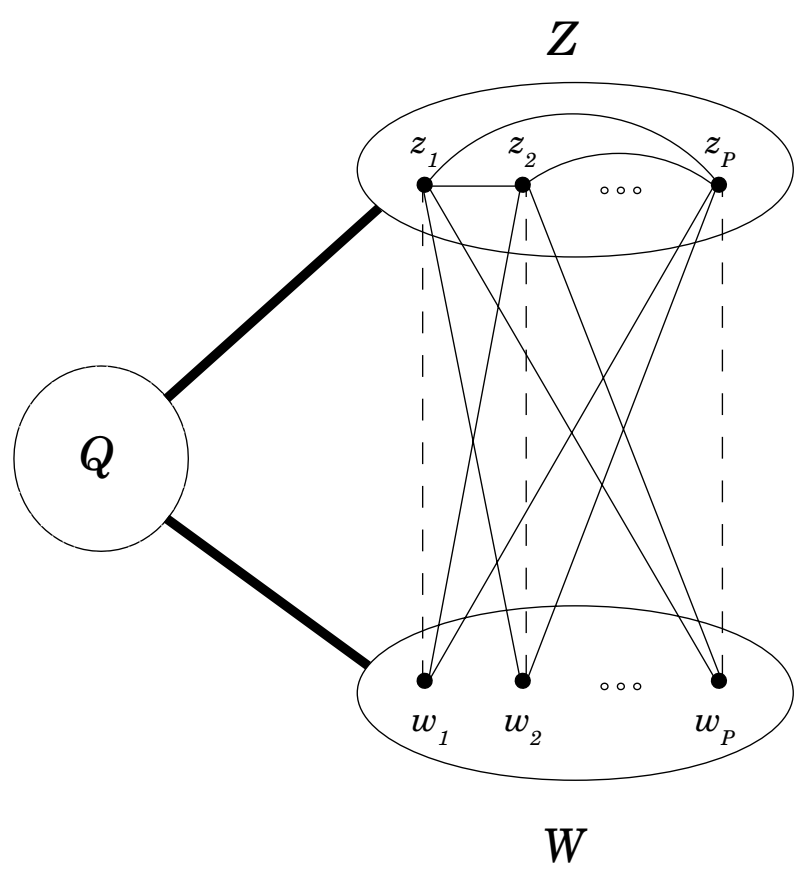

Figure 6. Graph $G_{p, q}$

distinct $q$ and $t$, the classes of graphs $(p, q)$-clique-Helly and $(p, t)$-clique-Helly are incomparable.

The following theorem describes a class of $(p, q)$ clique-Helly graphs.

Theorem 7.1 [30] Let $p, r>1, q>0$ such that $p+q \geq$ $r$. If $G$ is a $K_{r}$-free graph, then $G$ is $(p, q)$-clique-Helly.

Our aim is now to characterize $(p, q)$-clique-Helly graphs. We divide the characterization in two cases, the first deals with $p=1$.

Theorem 7.2 [30] Let $G$ be a graph, and let $W$ be the union of the $q^{+}$-cliques of $G$. Then $G$ is a $(1, q)$-cliqueHelly graph if and only if $G[W]$ contains $q$ universal vertices.

The second case corresponds to $p \geq 2$ and we employ some additional definitions.

Let $G$ be a graph and $C$ a $p$-complete set of $G$. The p-expansion relative to $C$ is the subgraph of $G$ induced by the vertices $w$ such that $w$ is adjacent to at least $p-1$ vertices of $C$.

We remark that the $p$-expansion for $p=3$ has been used for characterizing clique-Helly graphs $[35,83]$. It is clear that constructing a $p$-expansion relative to a given $p$-complete set can be done in polynomial time.

Let $\mathscr{F}$ be a partial hypergraph of $\mathcal{C}(G)$. The clique subgraph induced by $\mathscr{F}$ in $G$, denoted by $G_{c}[\mathscr{F}]$, is the subgraph of $G$ formed exactly by the vertices and edges belonging to the cliques of $\mathscr{F}$.

Lemma 7.1 Let $G$ be a graph, $C$ a p-complete set of it, $H$ the p-expansion of $G$ relative to $C$, and $\mathscr{C}$ the partial hypergraph $\mathcal{C}(G)$ formed by the cliques that contain at least $p-1$ vertices of $C$. Then $G_{c}[\mathscr{C}]$ is a spanning subgraph of $H$.

Let $G$ be a graph. The graph $\Phi_{q}(G)$ is defined as follows. The vertices of $\Phi_{q}(G)$ correspond to the $q$-complete sets of $G$, two vertices being adjacent in $\Phi_{q}(G)$ if the corresponding $q$-complete sets in $G$ are contained in a common clique. As an example see Figure 8.

We remark that $\Phi_{q}$ is precisely the operator $\Phi_{q, 2 q}$ described in [77], p.136, and the graph $\Phi_{2}(G)$ is the edge clique graph of $G$, introduced in [1].

An interesting property of $\Phi_{q}$ is that it preserves the $q^{+}$-cliques of $G$, that is, every $q^{+}$-clique of $G$ is a clique of $\Phi_{q}(G)$, and vice versa. Then, given a $q^{+}$-clique $C$ of $G$, denote by $\varphi_{q}(C)$ the clique of $\Phi_{q}(G)$ associated to $C$.

Let $G$ be a graph and $\mathcal{C}(G)$ its clique hypergraph. Let $\mathscr{F}$ be a partial hypergraph of $\mathcal{C}(G)$ containing some $q^{+}$. cliques of $G$. Define $\varphi_{q}(\mathscr{F})$ to be the set of cliques $\left\{\varphi_{q}(C): C \in E(\mathscr{F})\right\}$. If $\mathscr{C}$ is a partial hypergraph of $\mathcal{C}\left(\Phi_{q}(G)\right)$, define $\varphi_{q}^{-1}(\mathscr{C})$ as the set of cliques $\left\{\varphi_{q}^{-1}(C): C \in E(\mathscr{C})\right\}$. As a consequence we have:

Corollary 7.1 Let $G$ be a graph, $\mathscr{F}$ a partial hypergraph of $\mathcal{C}(G)$, containing some $q^{+}$-cliques of $G$, and $\mathscr{C}$, such that $E(\mathscr{C})=\varphi_{q}(\mathscr{F})$. Then $\mid$ core $(\mathscr{F}) \mid \geq q$ if and only if $|\operatorname{core}(\mathscr{C})| \geq 1$.

Lemma 7.2 Let $C$ be a $(p+1)$-complete set of a graph $G$, and let $\mathscr{C}$ be a partial $p^{-}$-hypergraph of $\mathcal{C}(G)$ such that every clique of $\mathscr{C}$ contains at least $p$ vertices of $C$. Then $\operatorname{core}(\mathscr{C}) \neq \varnothing$.

The next result is a characterization of $(2,2)$-cliqueHelly graphs.

Theorem 7.3 [25] A graph $G$ is (2,2)-clique-Helly if and only if every extended triangle of $\Phi_{2}(G)$ contains a universal vertex.

Problem 7.1 ((2,2)-CliQUe-Helly GRAPH): Given a graph $G$, decide whether $G$ is $(2,2)$-clique-Helly. 
$G$
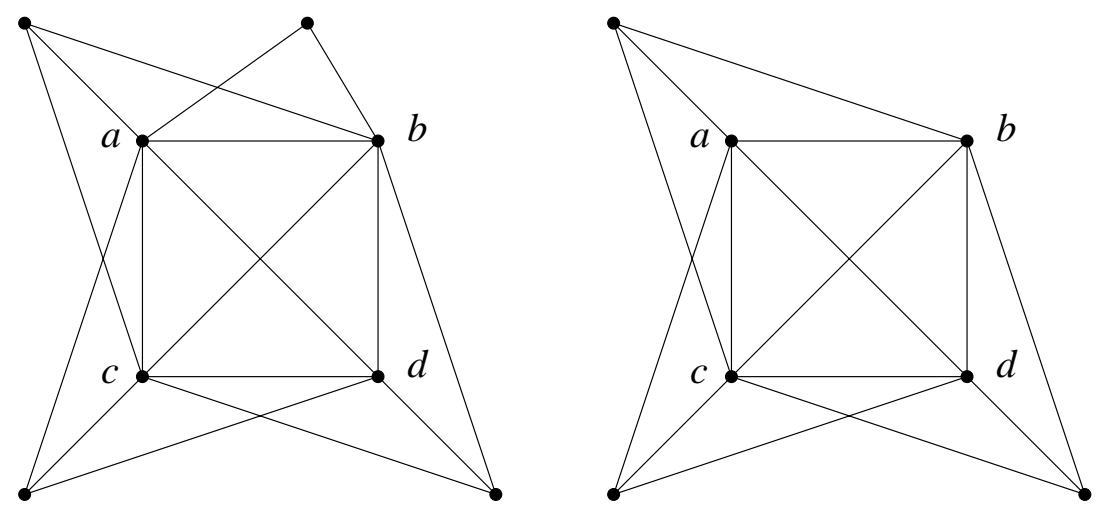

Figure 7. A graph $G$ and the 4-expansion relative to $\{a, b, c, d\}$.

$G$

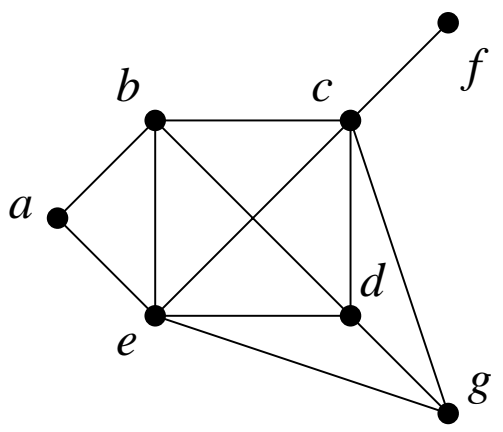

$\Phi_{3}(G)$

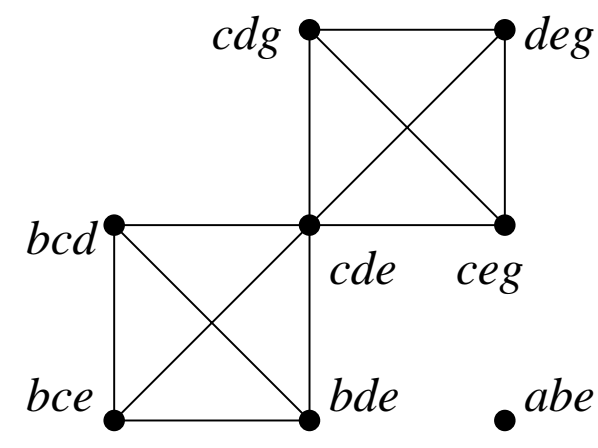

Figure 8. A graph $G$ and $\Phi_{3}(G)$ 
Algorithm 7.1 (RECOGNIZING $(2,2)$-CLIQUe-Helly GRAPHS): To construct $\Phi_{2}(G)$ create one vertex for each edge of $G$. Then join two vertices by an edge if the corresponding edges are contained in a same clique of $G$.

Next, for every triangle $T$ of $\Phi_{2}(G)$, we construct the extended triangle of $T$ and verify if it contains a universal vertex. If we find one extended triangle which does not have a universal vertex, then we stop as $G$ is not $(2,2)$ clique-Helly, otherwise it is.

In order to calculate the complexity of this algorithm, first note that the number of vertices of $\Phi_{2}(G)$ is $m$. The time complexity to construct $\Phi_{2}(G)$ is $O\left(m^{2}\right)$, and to verify if there exists one extended triangle without universal vertex is $O\left(\mathrm{~m}^{5}\right)$. Therefore one can verify if a graph is $(2,2)$-clique-Helly in time $O\left(m^{5}\right)$.

Now we present a generalization of this result.

Theorem 7.4 [30] Let $p>1$ be an integer. Then a graph $G$ is $(p, q)$-clique-Helly if and only if every $(p+1)$ expansion of $\Phi_{q}(G)$ contains a universal vertex.

Proof. Suppose that $G$ is a $(p, q)$-clique-Helly graph and there exists a $(p+1)$-expansion $T$, relative to a $(p+1)$ complete set $C$ of $\Phi_{q}(G)$, such that $T$ contains no universal vertex.

Denote $H=\Phi_{q}(G)$. Let $\mathscr{C}$ be the partial hypergraph $\mathcal{C}(H)$ that contains at least $p$ vertices of $C$. Consider a partial $p^{-}$-hypergraph $\mathscr{C}^{\prime}$ of $\mathscr{C}$. By Lemma 7.2, $\operatorname{core}\left(\mathscr{C}^{\prime}\right) \neq \varnothing$. This implies that $\mathscr{C}$ is $(p, 1)$-intersecting. By Corollary 7.1, $\mathscr{F}=\varphi_{q}^{-1}(\mathscr{C})$ is $(p, q)$-intersecting. Since $G$ is $(p, q)$-clique-Helly, we conclude that $\mathscr{F}$ has a $q^{+}$-core. By using Corollary 7.1 again, $\mathscr{C}$ has an $1^{+}$core, which means that $H_{c}[\mathscr{C}]$ contains a universal vertex. Moreover, by Lemma 7.1, $H_{c}[\mathscr{C}]$ is a spanning subgraph of $T$. However, $T$ contains no universal vertex. This is a contradiction. Therefore, every $(p+1)$-expansion of $H$ contains a universal vertex.

Conversely, assume by contradiction that $G$ is not $(p, q)$-clique-Helly. Let $\mathscr{F}$ be a minimal $(p, q)$ intersecting partial hypergraph of $\mathcal{C}(G)$ which does not have a $q^{+}$-core. Denote $E(\mathscr{F})=\left\{C_{1}, \ldots, C_{k}\right\}, C_{i} \in$ $\mathcal{C}(G)$. Clearly, $k>p$.

The minimality of $\mathscr{F}$ implies that there exists a $q$ subset $Q_{i} \subseteq \operatorname{core}\left(\mathscr{F}-C_{i}\right)$, for $i=1, \ldots, k$. It is clear that $Q_{i} \nsubseteq C_{i}$. Moreover, every two distinct $Q_{i}, Q_{j}$ are contained in a common clique, since $k \geq 3$. Hence the sets $Q_{1}, Q_{2}, \ldots, Q_{p+1}$ correspond to a $(p+1)$-complete set $C$ in $\Phi_{q}(G)$.

Let $\mathscr{C}^{\prime}$ be the partial hypergraph of $\mathcal{C}(H)$ formed by the cliques that contain at least $p$ vertices of $C$. Let $\mathscr{C}=\varphi_{q}(\mathscr{F})$. Since every $C_{i} \in E(\mathscr{F})$ contains at least $p$ sets from $Q_{1}, Q_{2}, \ldots, Q_{p+1}$, it is clear that the clique $\varphi_{q}\left(C_{i}\right) \in E(\mathscr{C})$ contains at least $p$ vertices of $C$. Therefore, $\varphi_{q}\left(C_{i}\right) \in E\left(\mathscr{C}^{\prime}\right)$, for $i=1, \ldots, k$.

Let $T$ be the $(p+1)$-expansion of $H$ relative to $C$. By Lemma 7.1, $H_{c}\left[\mathscr{C}^{\prime}\right]$ is a spanning subgraph of $T$. Therefore, $Q \subseteq V(T)$, for every $Q \in E\left(\mathscr{C}^{\prime}\right)$. In particular, $V\left(\varphi_{q}\left(C_{i}\right)\right) \subseteq V(T)$, for $i=1, \ldots, k$. By hypothesis, $T$ contains a universal vertex $x$. Then $x$ is adjacent to all the vertices of $\varphi_{q}\left(C_{i}\right) \backslash\{x\}$, for $i=1, \ldots, k$. This implies that $\varphi_{q}\left(C_{i}\right)$ contains $x$, otherwise $\varphi_{q}\left(C_{i}\right)$ would not be maximal. Thus, $\operatorname{core}(\mathscr{C}) \neq \varnothing$. By Corollary 7.1, $\mathscr{F}$ has a $q^{+}$-core, a contradiction. Hence, $G$ is a $(p, q)$-cliqueHelly graph.

From the above theorem one can recognize $(p, q)$ clique-Helly graphs in polynomial time if $p$ and $q$ are fixed.

Problem 7.2 $((p, q)$-Clique-Helly GRAPH $)$ : Let $p, q \geq 1$ be fixed integers. Given a graph $G$, decide whether $G$ is $(p, q)$-clique-Helly.

We present now the algorithm, for the case $p \geq 2$. Let $G$ be a graph.

Algorithm 7.2 (RECOGNIZING $(p, q)$-CLIQUe-Helly GRAPHS): Construct the graph $\Phi_{q}(G)$, having as vertices the p-complete sets of $G$, and making two vertices adjacent when the corresponging p-complete sets are both contained in a same clique.

Next, for every $(p+1)$-complete set $C$ of $\Phi_{q}(G)$, we construct the $(p+1)$-expansion relative to $C$ and verify if it contains a universal vertex. If we find $a(p+1)$ expansion which does not have a universal vertex, then we stop as $G$ is not $(p, q)$-clique-Helly, otherwise it is.

In order to calculate the complexity of this algorithm, first note that the number of vertices of $\Phi_{q}(G)$ is $t=O\left(n^{q}\right)$. The time complexity to construct $\Phi_{q}(G)$ is $O\left(n^{2 q} q^{2}\right)$, whereas to verify if there exists a $(p+1)$ expansion with no universal vertex is $O\left(t^{p+1} m^{\prime}\right)=$ $O\left(n^{q(p+1)} m^{\prime}\right)$, where $m^{\prime}=O\left(t^{2}\right)$ is the number of edges of $\Phi_{q}(G)$. Therefore one can verify if a graph is $(p, q)$ clique-Helly in $O\left(n^{q(p+3)}\right)$ time.

If $p$ or $q$ is variable, this procedure does not lead to a polynomial time algorithm. Indeed, the problem is NPhard in both cases.

Problem $7.3((p, q)$-CLIQUe-HELlY GRAPH, $q$ VARI$\mathrm{ABLE})$ : Let $p$ be a fixed positive integer. Given a graph $G$ and a positive integer $q$, decide whether $G$ is $(p, q)$ clique-Helly. 
Theorem 7.5 [30] $(p, q)$-CliQue-Helly GRAPH, $q$ VARIABLE is NP-hard.

Problem 7.4 $((p, q)$-CLIQUE-HELLY GRAPH, $p$ VARIABLE): Let $q$ be a fixed positive integer. Given a graph $G$ and a positive integer $p$, decide whether $G$ is $(p, q)$ clique-Helly.

Theorem 7.6 [30] $(p, q)$-CLIQUE-Helly GRAPH, $p$ VARIABLE is NP-hard.

\subsection{Helly defect}

For any clique-Helly graph, its clique graph is also clique-Helly [44]. However, if a graph is not clique-Helly it is still possible for its clique graph to be clique-Helly. This motivated the definition of Helly defect [8], a parameter that informs how many times the clique operator must be applied for a graph to become clique-Helly. The Helly defect of a graph $G$ is the smallest $i$ such that $K^{i}(G)$ is clique-Helly. There are graphs with any desired finite Helly defect [18]. However if $K^{i}(G)$ is not clique-Helly, for any finite $i$, we say that its Helly defect is infinite. Trivially, the Helly defect of a clique-Helly graph is 0 , while that of a divergent graph is infinity.

Problem 7.5 (Helly DefeCt ONE): Given a graph $G$, decide whether the Helly defect of $G$ is at most one.

The Helly defect of a graph is less than or equal to 1 when it or its clique graph is clique-Helly. In fact, this problem corresponds to the case $q=1$ if one asks if, for a given fixed $q$, the graph or its clique graph is $(2, q)$-cliqueHelly.

Problem 7.6 (CliQue GRAPH IS $(2, q)$-CliQueHELly): Let $q \geq 1$ be a fixed integer. Given a graph $G$, decide whether $K(G)$ is $(2, q)$-clique-Helly.

Theorem 7.7 [29] Clique GRAPH IS $(2, q)$-CliQueHELlY is NP-hard.

Corollary 7.2 [29] HeLLY DEFECT ONE is NP-hard.

\section{Hereditary Helly Property}

A hypergraph is strong Helly if for every partial hypergraph $\mathcal{H}^{\prime}$ of $\mathcal{H}$, there exist two hyperedges in $\mathcal{H}^{\prime}$ whose core is equal to the core of $\mathcal{H}^{\prime}$. A hypergraph $\mathcal{H}$ is hereditary Helly if all subhypergraphs of $\mathcal{H}$ are Helly. In this section we present algorithms and characterizations on generalizations of these two variants of the Helly property.
In fact, we show that these two concepts are equivalent. First we characterize hereditary $p$-Helly hypergraphs and then consider the hereditary Helly property applied to special families of vertices of a graph, such as cliques, disks, bicliques, open and closed neighbourhoods.

\subsection{Hypergraphs}

Since the number of partial hypergraphs and of subhypergraphs of a given hypergraph can be exponential in the size of the hypergraph, the definitions do not lead directly to algorithms to verify, in polynomial time, if a hypergraph is strong Helly or hereditary Helly.

Problem 8.1 (Hereditary Helly hypergraph): Decide whether a hypergraph is hereditary Helly.

In [92] it has been shown that a hypergraph $\mathcal{H}$ is strong Helly if and only if for every three hyperedges of $\mathcal{H}$ there exist two of them whose core equals the core of the three hyperedges. This characterization leads to an algorithm for recognizing strong Helly hypergraphs with time complexity $O\left(\mathrm{rm}^{3}\right)$, where $r$ and $m$ are, respectively, the rank and the number of hyperedges of the hypergraph.

In [23] it was presented an algorithm for recognizing hereditary Helly hypergraphs that needs $O\left(m \Delta r^{4}\right)$ time and $O\left(m r^{2}\right)$ space, where $\Delta$ is the maximum degree of the hypergraph.

Generalizing these concepts, it follows that a hypergraph $\mathcal{H}$ is strong $p$-Helly if for every partial $(p+1)^{+}$ hypergraph $\mathcal{H}^{\prime}$ of $\mathcal{H}$, there exist $p$ hyperedges in $\mathcal{H}^{\prime}$ whose core equals the core of $\mathcal{H}^{\prime}$. Also, a hypergraph $\mathcal{H}$ is hereditary $p$-Helly if all subhypergraphs of $\mathcal{H}$ are $p$-Helly.

Theorem 8.1 [49]

(i) A hypergraph in which every hyperedge is a set of edges of some path of a tree is strong 3-Helly.

(ii) A hypergraph in which every hyperedge is a set of edges of some subtree of a tree with $k$ leaves is strong $k$-Helly.

The following theorem characterizes strong $p$-Helly and hereditary $p$-Helly hypergraphs. It implies that they are equivalent.

Theorem 8.2 [34] The following statements are equivalent for a hypergraph $\mathcal{H}$ :

(i) $\mathcal{H}$ is strong p-Helly;

(ii) $\mathcal{H}$ is hereditary $p$-Helly;

(iii) $\mathcal{H}$ is $(p, q)$-Helly, for every $q$; 
(iv) every partial $(p+1)$-hypergraph of $\mathcal{H}$ is $(p, q)$-Helly for every $q$;

(v) there is no subhypergraph of $\mathcal{H}$ having a partial hypergraph isomorphic to $K_{p+1}^{p}$.

Proof. $(i) \Rightarrow(i i)$ Suppose that $\mathcal{H}$ contains a subhypergraph $\mathcal{H}^{\prime}$ that is not $p$-Helly. Let $\mathcal{H}^{\prime \prime}$ be a partial hypergraph of $\mathcal{H}^{\prime}$ which is $p$-intersecting with an empty core. Define a partial hypergraph $\mathcal{H}_{1}$ of $\mathcal{H}$ choosing for every hyperedge $E^{\prime \prime} \in E\left(\mathcal{H}^{\prime \prime}\right)$ the hyperedge of $\mathcal{H}$ that originated it. Since any $p$ hyperedges of $\mathcal{H}^{\prime \prime}$ contain one vertex that is not in the core of $\mathcal{H}^{\prime \prime}$, the same is true for any $p$ hyperedges and the core of $\mathcal{H}_{1}$. Therefore $\mathcal{H}$ is not strong $p$-Helly.

(ii) $\Rightarrow$ (iii) Suppose that $\mathcal{H}$ is not $(p, q)$-Helly, for some $q$. Let $\mathcal{H}^{\prime}$ be a $(p, q)$-intersecting partial hypergraph of $\mathcal{H}$ without a $q^{+}$-core. Denote the core of $\mathcal{H}^{\prime}$ by $C^{\prime}$. Every hyperedge of $\mathcal{H}^{\prime}$ properly contains $C^{\prime}$ because it belongs to a $(p, q)$-intersecting partial hypergraph, and $C^{\prime}$ is a $(q-1)^{-}$-set. Hence, in the subhypergraph $\mathcal{H}_{1}^{\prime}$ of $\mathcal{H}$ induced by $V(\mathcal{H}) \backslash C^{\prime}$, there is one hyperedge for every hyperedge of $\mathcal{H}^{\prime}$. Consider the partial hypergraph $\mathcal{H}_{1}^{\prime \prime}$ of $\mathcal{H}_{1}^{\prime}$ formed by these hyperedges. Note that $\mathcal{H}_{1}^{\prime \prime}$ is $p$ intersecting and has an empty core. Therefore $\mathcal{H}_{1}^{\prime}$ is not $p$-Helly.

$($ iii $) \Rightarrow($ iv $)$ Trivial.

$(i v) \Rightarrow(v)$ Let $\mathcal{H}^{\prime}$ be a partial hypergraph of a subhypergraph of $\mathcal{H}$ isomorphic to $K_{p+1}^{p}$. Clearly, $\mathcal{H}^{\prime}$ is not $(p, 1)$-Helly. Moreover, there exists a partial $(p+1)$ hypergraph $\mathcal{H}^{\prime \prime}$ of $\mathcal{H}$ in which every hyperedge contains a different hyperedge of $\mathcal{H}^{\prime}$. Hence, if the core of $\mathcal{H}^{\prime \prime}$ has size $c$, we can say that $\mathcal{H}^{\prime \prime}$ is $(p, c+1)$-intersecting, that is, $\mathcal{H}^{\prime \prime}$ is not $(p, c+1)$-Helly.

$(v) \Rightarrow(i)$ Suppose that $\mathcal{H}$ is not strong $p$-Helly. Then there is a partial hypergraph $\mathcal{H}^{\prime}$ of $\mathcal{H}$ such that the core of every $p$ hyperedges of $\mathcal{H}^{\prime}$ properly contains $C^{\prime}=$ $\operatorname{core}\left(\mathcal{H}^{\prime}\right)$. Perfom the following process: if $\mathcal{H}^{\prime}$ contains a hyperedge $E^{\prime}$ such that the core of $\mathcal{H}^{\prime}-E^{\prime}$ is $C^{\prime}$, redefine $E\left(\mathcal{H}^{\prime}\right)=E\left(\mathcal{H}^{\prime}\right) \backslash\left\{E^{\prime}\right\}$, and repeat; otherwise, stop.

After completion, observe that for any $E_{k} \in E\left(\mathcal{H}^{\prime}\right)$ there is a vertex $v_{k} \notin E_{k}$ in the core of $\mathcal{H}^{\prime}-E_{k}$. This means that the subhypergraph of $\mathcal{H}$ induced by $\left\{v_{1}, v_{2}, \ldots, v_{p+1}\right\}$ has a partial hypergraph isomorphic to the hypergraph formed by all $p$-subsets of a $(p+1)$-set.

We can apply the equivalence (i)-(iv) in order to formulate an algorithm for recognizing strong $p$-Helly graphs, as follows. First observe that the affirmative (iv) is equivalent to state that for every $(p+1)$-hypergraph $\mathcal{H}^{\prime}$ of $\mathcal{H}$ there exist $p$ hyperedges with the same core as $\mathcal{H}^{\prime}$.
Problem 8.2 (HEREDITARY $p$-HELly hyPERGRAPH): Let $p \geq 2$ be a fixed integer. Given a hypergraph $\mathcal{H}$, decide whether $\mathcal{H}$ is strong p-Helly.

Algorithm 8.1 (RECOGNIZING HEREDITARY $p$-HELlY HYPERGRAPHS): For every partial $(p+1)$-hypergraph of $\mathcal{H}$, compute its core and the core of every partial $p$ hypergraph of it. If every partial $(p+1)$-hypergraph $\mathcal{H}^{\prime}$ of $\mathcal{H}$ has a partial p-hypergraph whose core equals the core of $\mathcal{H}^{\prime}$, then $\mathcal{H}$ is strong $p$-Helly, otherwise it is not.

Computing the cores of a $(p+1)$-hypergraph and all its partial $p$-hypergraphs can be done in $O\left(p^{2} r\right)$ steps. Since there are $O\left(m^{p+1}\right)$ partial $(p+1)$-hypergraphs in a hypergraph, this algorithm has time complexity $O\left(p^{2} r m^{p+1}\right)$. For fixed $p$, the above algorithm terminates within polynomial time. The following theorem refers to $p$ variable.

Problem 8.3 (Hereditary $p$-Helly hypergraph, $p$ VARIABLE): Given a hypergraph $\mathcal{H}$ and an integer $p \geq 2$, decide whether $\mathcal{H}$ is strong $p$-Helly.

Theorem 8.3 [34] Hereditary $p$-Helly hyPERGRAPH, $p$ VARIABLE is co-NP-complete.

\subsection{Cliques of graphs}

We say that a graph is strong p-clique-Helly if its clique hypergraph is strong $p$-Helly, and that it is hereditary $p$-clique-Helly if all induced subgraphs of it are $p$ clique-Helly. As usual, we write clique-Helly to mean 2-clique-Helly.

Since every $p$-clique-Helly graph is also $(p+1)$ clique-Helly, every hereditary $p$-clique-Helly graph is also hereditary $(p+1)$-clique-Helly. The following result says that the clique hypergraph of a intersection graph of a family of edge paths of a tree is strong 4-Helly.

Theorem 8.4 [49] If $G$ is an intersection graph of edge paths of a tree, then $G$ is strong 4-clique-Helly.

Next, we consider the question of characterizing hereditary $p$-clique-Helly graphs.

Theorem 8.2 is valid for general hypergraphs, and in particular for the clique hypergraph of a graph. However, since the number of cliques of a graph may be exponential in the size of the graph [71], it does not lead to a polynomial-time algorithm for recognizing strong $p$-clique-Helly graphs. Similarly, the algorithm for recognizing $p$-clique-Helly graphs is also not suitable for recognizing hereditary $p$-clique-Helly graphs because the number of induced subgraphs may also be exponential in the size of the graph. 

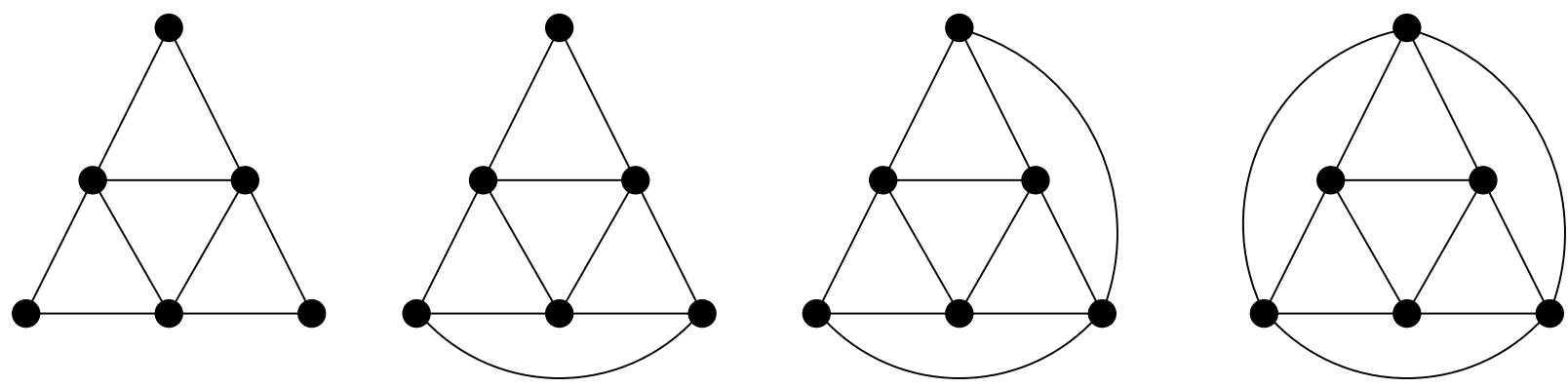

Figure 9. Forbidden subgraphs for hereditary clique-Helly graphs

The characterization of hereditary clique-Helly graphs given below uses the following definition. An edge $e$ of a triangle $T$ is good, relative to $T$, if any vertex adjacent to the vertices of $e$ is also adjacent to the other vertex of $T$.

Theorem 8.5 [92, 76] The following statements are equivalent for any graph $G$ :

(i) $G$ is hereditary clique-Helly;

(ii) $G$ is strong clique-Helly;

(iii) $G$ does not contain an ocular graph as an induced subgraph;

(iv) every triangle of $G$ has a good edge.

Figure 9 shows the ocular graphs.

Problem 8.4 (Hereditary CLIQUe-Helly GRAPH): Given a graph $G$, decide whether $G$ is hereditary cliqueHelly.

Algorithm 8.2 (RECOGNIZING HEREDITARY CLIQUEHELLY GRAPHS): For every triangle $T$ of $G$, verify if $T$ contains a good edge.

All the triangles of a graph can be computed in time $O(n m)$. We need $O(n)$ time to verify, for each one, if it contains a good edge. Therefore the complexity of the algorithm for recognizing hereditary clique-Helly graphs is $O\left(n^{2} m\right)$.

We can define the sandwich problem for hereditary clique-Helly graphs as we did in Section 3 for cliqueHelly graphs.

Problem 8.5 (Hereditary CLIQUe-Helly SANDWICH GRAPH): Given two graphs $G_{1}, G_{2}$ such that $G_{1} \subseteq G_{2}$, is there a sandwich graph for $G_{1}$ and $G_{2}$ which is hereditary clique-Helly?
Theorem 8.6 [28] Hereditary CLIQUE-Helly SANDWICH GRAPH is NP-complete.

For every integer $p \geq 3$, a graph $G$ is $p$-ocular if $V(G)$ is the union of the disjoint sets $W=\left\{w_{1}, w_{2}, \ldots, w_{p}\right\}$ and $U=\left\{u_{1}, u_{2}, \ldots, u_{p}\right\}$, where $W$ is a complete set, $U$ induces an arbitrary subgraph, and $w_{i}, u_{j}$ are adjacent precisely when $i \neq j$. The 3 -ocular graph corresponds to the ocular graph defined in [92]. A graph is p-ocular-free if it has not a $p$-ocular graph as an induced subgraph.

Lemma 8.1 [34] Any $(p+1)$-ocular graph is not $p$ clique-Helly, $p \geq 2$.

The following characterization of hereditary $p$-cliqueHelly graphs is a generalization of the one presented above for hereditary clique-Helly graphs. We need one more concept, which is a generalization of that of a good edge. A $p$-complete subset $C^{\prime}$ of a $(p+1)$-complete set $C$ is good, relative to $C$, if any vertex adjacent to all vertices of $C^{\prime}$ is also adjacent to the vertex in $C \backslash C^{\prime}$.

Theorem 8.7 [34] The following statements are equivalent for any graph $G$ :

(i) G is strong p-clique-Helly;

(ii) $G$ is hereditary p-clique-Helly;

(iii) $G$ is $(p+1)$-ocular-free;

(iv) every $(p+1)$-complete set of $G$ contains a good $p$ complete subset.

Problem 8.6 (HEREDITARY $p$-CLIQUE-HELLY GRAPH): Let $p \geq 2$ be a fixed integer. Given a graph $G$, decide whether $G$ is hereditary p-clique-Helly.

Algorithm 8.3 (RECOGNIZING HEREDITARY $p$ CLIQUE-HELLY GRAPHS): For every $(p+1)$-complete set $C$ of $G$, verify if $C$ contains a good p-complete set. 

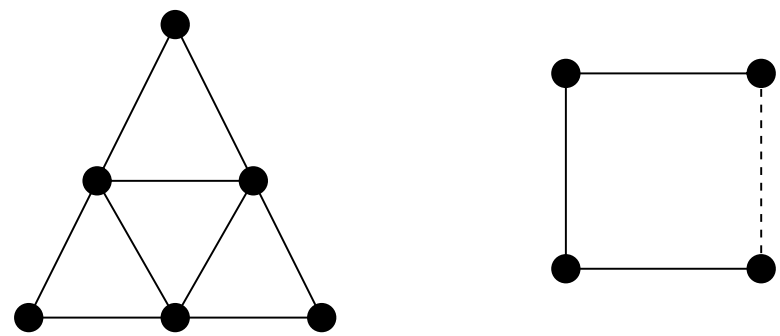

Figure 10. Forbidden subgraphs for hereditary disk-Helly graphs

The number of $(p+1)$-complete sets in a graph with $n$ vertices is $O\left(n^{p+1}\right)$. We need $O(n p)$ time to verify, for each one, if it contains a good $p$-complete set. Therefore the complexity of the above algorithm is $O\left(p n^{p+2}\right)$. For fixed $p$, the algorithm terminates within polynomial time. For $p$ variable, we have the following result.

Problem 8.7 (HEREDITARY $p$-CLIQUe-Helly GRAPH, $p$ VARIABLE): Given a graph $G$ and an integer $p \geq 2$, decide whether $G$ is hereditary p-clique-Helly.

Theorem 8.8 [34] HeREDitary $p$-CliQUe-Helly GRAPH, $p$ VARIABLE is NP-hard.

\subsection{Other Hereditary Helly Classes of Graphs}

A hereditary disk-Helly graph is a graph whose induced subgraphs are disk-Helly. Similarly, define hereditary biclique-Helly, hereditary open and closedneighbourhood-Helly graphs. The following theorems describe characterizations for these classes, in terms of forbidden induced subgraphs.

Problem 8.8 (HeREDitary DISK-HELly GRAPH): Given a graph $G$, decide whether $G$ is hereditary disk-Helly.

Theorem 8.9 [35] A graph is hereditary disk-Helly if and only if it does not contain the graphs of Figure 10, as induced subgraphs.

Problem 8.9 (HEREDITARY

BICLIQUE-HELLY GRAPH): Given a graph $G$, decide whether $G$ is hereditary biclique-Helly.

Theorem 8.10 [52] A graph is hereditary biclique-Helly if and only if it does not contain the graphs of Figure 11 as induced subgraphs.

Problem 8.10 (HEREDITARY OPEN NEIGHBOURHOODHELLY GRAPH): Given a graph $G$, decide whether $G$ is hereditary open neighbourhood-Helly.
Theorem 8.11 [52] A graph is hereditary open neighbourhood-Helly if and only if it does not contain the graphs of Figure 12 as induced subgraphs.

Problem 8.11 (HEREDITARY

CLOSED NEIGHBOURHOOD-HELLY GRAPH): Given a graph $G$, decide whether $G$ is hereditary closed neighbourhoodHelly.

Theorem 8.12 [52] A graph is hereditary closed neighbourhood-Helly if and only if it does not contain the graphs of Figure 13 as induced subgraphs.

It follows directly from the characterizations of the above considered classes that they can be recognized in polynomial time.

By comparing the above forbidden families, we can also conclude:

Corollary 8.1 Let $G$ be a graph with girth at least 7 . Then $G$ is hereditary clique-Helly, hereditary bicliqueHelly, hereditary open neighbourhood-Helly and hereditary closed neighbourhood-Helly.

\section{Summary of Results}

Table 1 summarizes the complexity results of the various algorithmic problems considered in the paper. The complexities expressed in terms of $O$-notation in the table correspond to straighforward algorithms realizing the associated characterizations.

\section{Proposed Problems}

To conclude, we propose the following problems.

1. [90] Describe a structural characterization for $(2, q)$ Helly hypergraphs.

2. Determine the complexity of recognizing $(p, q)$ Helly hypergraphs, for fixed $p$. In special, consider $p=2$.

3. Conjecture: "Every clique-Helly graph contains a vertex whose removal maintains it as a clique-Helly graph".

4. A graph $G$ is matching-Helly when the family of maximum matchings of $G$ is Helly. Characterize matching-Helly graphs.

5. Characterize $(p, q, s)$-clique-Helly graphs. 

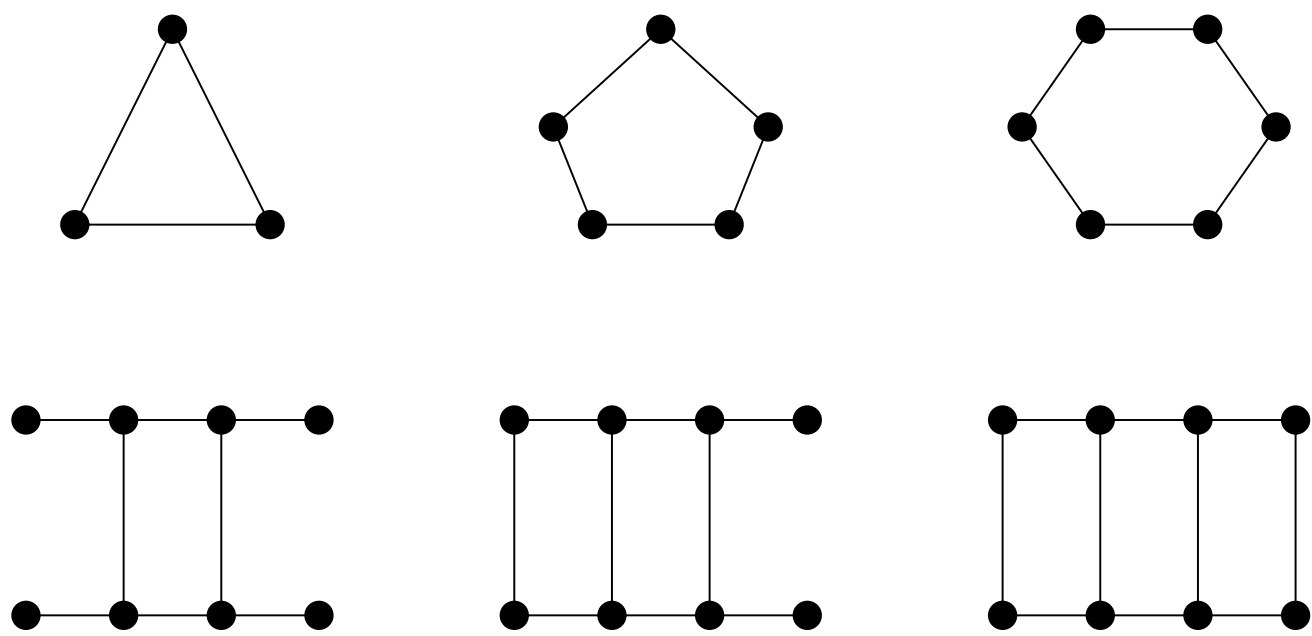

Figure 11. Forbidden subgraphs for hereditary biclique-Helly graphs
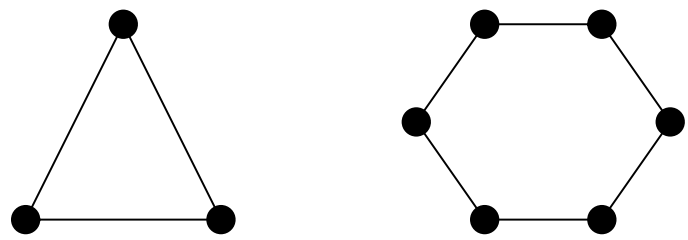

Figure 12. Forbidden subgraphs for hereditary open neighbourhood-Helly gragphs
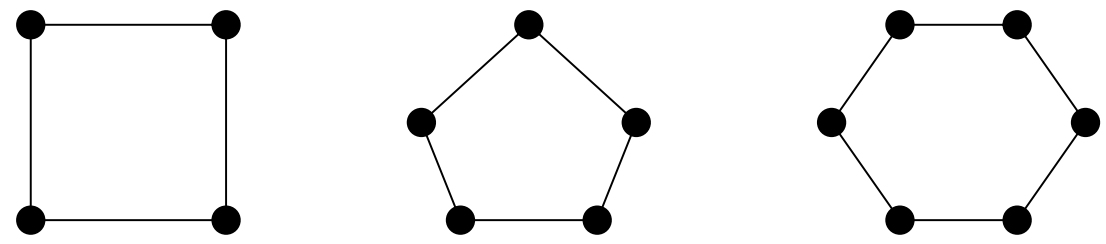

Figure 13. Forbidden subgraphs for hereditary closed neighbourhood-Helly graphs 


\begin{tabular}{|c|c|c|}
\hline Problem & Complexity & Reference \\
\hline 2.1 HELLY HYPERGRAPH & $O\left(n^{4} m\right)$ & [13] \\
\hline 3.1 CliQue-Helly GRAPH & $O\left(n m^{2}\right)$ & {$[35,83]$} \\
\hline 3.2 CliQue-HELlY SANDWICH & NP-complete & [28] \\
\hline 3.3 BICLIQUE-HELLY GRAPH & $O\left(n^{3} m\right)$ & [53] \\
\hline \multirow[t]{2}{*}{ 3.4 HELLY CIRCULAR-ARC GRAPH } & $O\left(n^{3}\right)$ & [48] \\
\hline & $O(n+m)$ & [65] \\
\hline 4.1 $p$-HELLY HYPERGRAPH & $O\left(n^{p+2} m\right)$ & [13] \\
\hline 4.2 LIST $p$-HELLY HYPERGRAPH & Co-NP-complete & [33] \\
\hline $5.1 k$-BOUNDED $p$-HELLY HYPERGRAPH & $O\left(n m^{k} k^{p}\right)$ & Algorithm 5.1 \\
\hline $5.2 k$-BOUNDED $p$-HELLY HYPERGRAPH, $k$ VARIABLE & Co-NP-complete & [31] \\
\hline $5.3 k$-BOUNDED $p$-CLIQUE-HELLY GRAPH & Co-NP-complete & [31] \\
\hline 5.4 PlANAR 3-BOUNDED CLIQUE-HELLY GRAPH & $O\left(n^{2}\right)$ & [3] \\
\hline $6.1(p, q)$-INTERSECTING HYPERGRAPH, $p$ VARIABLE & Co-NP-complete & [33] \\
\hline $6.2(p, q, s)$-HELLY HYPERGRAPH, $s$ VARIABLE & $O\left(n^{b(p+a+1)+1} m^{p}+n m^{p+a}\right)$ & [32] \\
\hline $6.3(p, q, s)$-HELLY HYPERGRAPH, $p$ VARIABLE & NP-hard & [33] \\
\hline $6.4(p, q, s)$-HELLY HYPERGRAPH, $q$ VARIABLE & Co-NP-complete & [33] \\
\hline $6.6(2, q)$-HELLY HYPERGRAPH, $q$ FIXED & $O\left(n^{3 q+1} m\right)$ & [33] \\
\hline \multirow[t]{2}{*}{$6.7(2, q)$-HELLY HYPERGRAPH, $r-q$ FIXED } & $O\left(n m^{r-q+4}\right)$ & [90] \\
\hline & $O\left(n^{r-q+2} m^{3}\right)$ & Algorithm 6.3 \\
\hline $7.1(2,2)$-CLIQUE-HELLY GRAPH & $O\left(m^{5}\right)$ & {$[25]$} \\
\hline $7.2(p, q)$-CLIQUE-HELLY GRAPH & $O\left(n^{q(p+3)}\right)$ & {$[30]$} \\
\hline $7.3(p, q)$-CliQue-Helly GRAPH, $q$ VARIABLE & NP-hard & {$[30]$} \\
\hline $7.4(p, q)$-Clique-Helly GRAPH, $p$ VARIABle & NP-hard & {$[30]$} \\
\hline 7.5 HELLY DEFECT & NP-hard & [29] \\
\hline \multirow[t]{2}{*}{ 8.1 HEREDitaRy HELLY HYPERGRAPH } & $O\left(r^{3}\right)$ & [92] \\
\hline & $O\left(r^{4} m^{2}\right)$ & [23] \\
\hline 8.2 HEREDITARY $p$-HELLY HYPERGRAPH & $O\left(r m^{p+1}\right)$ & [34] \\
\hline 8.3 HEREDITARY $p$-HELLY HYPERGRAPH, $p$ VARIABLE & Co-NP-complete & [34] \\
\hline 8.4 HEREDITARY CLIQUE-HELLY GRAPH & $O\left(n^{2} m\right)$ & {$[76,92]$} \\
\hline 8.5 HEREDITARY CLIQUE-HELLY SANDWICH GRAPH & NP-complete & {$[28]$} \\
\hline 8.6 HEREDITARY $p$-CLIQUE-HELLY GRAPH & $O\left(n^{p+2}\right)$ & [34] \\
\hline 8.7 HEREDITARY $p$-CLIQUE-HELLY GRAPH, $p$ VARIABLE & NP-hard & [34] \\
\hline 8.8 HEREDITARY DISK-HELLY GRAPH & $O\left(n^{2} m\right)$ & [35] \\
\hline 8.9 HEREDITARY BICLIQUE-HELLY GRAPH & $O\left(n^{2} m^{3}\right)$ & {$[52]$} \\
\hline 8.10 HEREDITARY OPEN NEIGHBORHOOD-HELLY GRAPH & $O\left(n^{2} m^{2}\right)$ & {$[52]$} \\
\hline 8.11 HEREDITARY CLOSED NEIGHBORHOOD-HELLY GRAPH & $O\left(n^{2} m^{2}\right)$ & {$[52]$} \\
\hline
\end{tabular}

Table 1 . Summary of complexity results 
6. Determine the complexity of recognizing $(p, q, s)$ clique-Helly graphs, for fixed $p, q$.

7. Is there an algorithm to decide if the Helly defect of a graph $G$ is finite?

\section{References}

[1] M. O. Albertson and K. L. Collins. Duality and perfection for edges in cliques. Journal of Combinatorial Theory, Series B, 36:298-309, 1984.

[2] L. Alcón, L. Faria, C. M. H. Figueiredo, and M. Gutierrez. Clique graphs is NP-complete. Manuscript, 2006.

[3] L. Alcón and M. Gutierrez. Cliques and extended triangles. A necessary condition for planar clique graphs. Discrete Applied Mathematics, 141:3-17, 2004.

[4] N. Alon and D. Kleitman. Piercing convex sets and the Hadwiger-Debrunner (p,q)-problem. Advances in Mathematics, 96:103-112, 1992.

[5] N. Amenta. Helly theorems and generalized linear programming. In Symposium on Computational Geometry, pages 63-72, 1993.

[6] H-J Bandelt, M. Farber, and P. Hell. Absolute reflexive retracts and absolute bipartite retracts. Discrete Applied Mathematics, 44(1-3):9-20, 1993.

[7] H. J. Bandelt and E. Pesch. Dismantling absolute retracts of reflexive graphs. European Journal of Combinatorics, 10:210-220, 1989.

[8] H-J Bandelt and E. Pesch. Efficient characterizations of $n$-chromatic absolute retracts. Journal on Combinatorial Theory Series B, 53:5-31, 1991.

[9] A. Barg, G. Cohen, S. Encheva, G. Kabatiansky, and G. Zémor. A hypergraph approach to the identifying parent property: The case of multiple parents. SIAM Journal on Discrete Mathematics, 14(3):423431, 2001.

[10] M. Benke. Efficient type reconstruction in the presence of inheritance. In A. M. Borzyszkowski and S. Sokolowski, editors, Proceedings of Mathematical Foundations of Computer Science (MFCS '93), volume 711 of $L N C S$, pages 272-280, Berlin, Germany, 1993. Springer.
[11] C. Berge. Graphes et Hypergraphes. Dunod, Paris, 1970. (Graphs and Hypergraphs, North-Holland, Amsterdam, 1973, revised translation).

[12] C. Berge. Hypergraphs. Gauthier-Villars, Paris, 1987.

[13] C. Berge and P. Duchet. A generalization of Gilmore's theorem. In M. Fiedler, editor, Recent Advances in Graph Theory, pages 49-55. Acad. Praha, Prague, 1975.

[14] B. Bollobás. Combinatorics. Cambridge University Press, Cambridge, 1986.

[15] A. Bondy, G. Durán, M. C. Lin, and J. L. Szwarcfiter. Self-clique graphs and matrix permutations. $J$. Graph Theory, 44(3):178-192, 2003.

[16] F. Bonomo. Self-clique Helly circular-arc graphs. Discrete Mathematics, 306:595-597, 2006.

[17] F. Bonomo, M. Chudnovski, and G. Durán. Partial characterizations of clique-perfect graphs. Eletronic Notes on Discrete Mathematics, 19:95-101, 2005.

[18] C. F. Bornstein and J. L. Szwarcfiter. On clique convergent graphs. Graphs and Combinatorics, 11:213-220, 1995.

[19] A. Brandstädt, V. Chepoi, and F. Dragan. The algorithmic use of hypertree structure and maximum neighbourhood orderings. Discrete Applied Mathematics, 82(1-3):43-77, 1998.

[20] A. Brandstädt, V. Chepoi, F. Dragan, and V. Voloshin. Dually chordal graphs. SIAM Journal on Discrete Mathematics, 11(3):437-455, aug 1998.

[21] A. Brandstädt, V. B. Le, and J. P. Spinrad. Graph classes: A survey. SIAM Monographs on Discrete Mathematics and Applications. Society for Industrial and Applied Mathematics, Philadelphia, PA, 1999.

[22] A. Bretto, J. Azema, H. Cherifi, and B. Laget. Combinatorics and image processing. Grafical Models and Image Processing, 59(5):265-277, 1997.

[23] A. Bretto, S. Ubéda, and J. Žerovnik. A polynomial algorithm for the strong Helly property. Information Processing Letters, 81:55-57, 2002.

[24] P. L. Butzer, R. J. Nessel, and E. L. Stark. Eduard Helly (1884-1943): In memoriam, volume 7. Resultate der Mathematik, 1984. 
[25] M. R. Cerioli. Edge-clique Graphs (in portuguese). Ph.D. Thesis, COPPE - Sistemas, Universidade Federal do Rio de Janeiro, Rio de Janeiro, 1999.

[26] S. A. Cook. The complexity of theorem-proving procedures. Proc. 3rd Ann. ACM Symp. on Theory of Computing Machinery, New York, pages 151-158, 1971.

[27] L. Danzer, B. Grünbaum, and V. L. Klee. Helly's theorem and its relatives. In Proc. Symp. on Pure Math AMS, volume 7, pages 101-180, 1963.

[28] M. C. Dourado, P. Petito, and R. B. Teixeira. Helly property and sandwich graphs. In Proceedings of ICGT 2005, volume 22 of Eletronic Notes in Discrete Mathematics, pages 497-500. Elsevier B.V., 2005 .

[29] M. C. Dourado, F. Protti, and J. L. Szwarcfiter. On the Helly defect of a graph. Journal of the Brazilian Computer Society, 7(3):48-52, 2001.

[30] M. C. Dourado, F. Protti, and J. L. Szwarcfiter. Characterization and recognition of generalized cliqueHelly graphs. In J. Hromkovič, M. Nagl, and B. Westfechtel, editors, Proceedings WG 2004, volume 3353 of Lecture Notes in Computer Science, pages 344-354. Springer-Verlag, 2004.

[31] M. C. Dourado, F. Protti, and J. L. Szwarcfiter. The Helly property on subfamilies of limited size. Information Processing Letters, 93:53-56, 2005.

[32] M. C. Dourado, F. Protti, and J. L. Szwarcfiter. On Helly hypergraphs with predescribed intersection sizes. Submitted, 2005.

[33] M. C. Dourado, F. Protti, and J. L. Szwarcfiter. Complexity aspects of generalized Helly hypergraphs. Information Processing Letters, 99:13-18, 2006.

[34] M. C. Dourado, F. Protti, and J. L. Szwarcfiter. On the strong $p$-Helly property. Discrete Applied Mathematics, to appear, 2006.

[35] F. F. Dragan. Centers of Graphs and the Helly Property (in russian). Ph. D. Thesis, Moldava State University, Chisinău, 1989.

[36] F. F. Dragan, C. F. Prisacaru, and V. D. Chepoi. Location problems in graphs and the Helly property. Diskretnája Matematica, 1992.
[37] P. Duchet. Proprieté de Helly et problèmes de représentations. In Colloquium International CNRS 260, Problèmes Combinatoires et Théorie de Graphs, pages 117-118, Orsay, France, 1976. CNRS.

[38] P. Duchet. Hypergraphs. In R. L. Graham, M. Grötschel, and L. Lovász, editors, Handbook of Combinatorics, volume 1, pages 381432, Amsterdam-New York-Oxford, 1995. Elsevier North-Holland.

[39] P. Duchet and H. Meyniel. Ensembles convexes dans les graphes. I: Théorèmes de Helly et de Radon pour graphes et surfaces. European Journal of Combinatorics, 4:127-132, 1983.

[40] G. Durán. Some new results on circle graphs. Matemática Contemporânea, 25:91-106, 2003.

[41] G. Durán, A. Gravano, M. Groshaus, F. Protti, and J. L. Szwarcfiter. On a conjecture concerning Helly circle graphs. Pesquisa Operacional, pages 221229, 2003.

[42] G. Duran and M. C. Lin. Clique graphs of helly circular arc graphs. Ars Combinatoria, 60:255-271, 2001.

[43] J. Eckhoff. Helly, Radon, and Carathéodory type theorems. In Handbook of Convex Geometry, pages 389-448. North-Holland, 1993.

[44] F. Escalante. Über iterierte clique-graphen. Abhandlungender Mathematischen Seminar der Universität Hamburg, 39:59-68, 1973.

[45] R. Fagin. Acyclic database schemes of various degrees: A painless introduction. In G. Ausiello and M. Protasi, editors, Proceedings of the 8th Colloquium on Trees in Algebra and Programming (CAAP'83), volume 159 of LNCS, pages 65-89, L'Aquila, Italy, mar 1983. Springer.

[46] R. Fagin. Degrees of acyclicity for hypergraphs and relational database systems. Journal of the Association for Computing Machinery, 30:514-550, 1983.

[47] C. Flament. Hypergraphes arborés. Discrete Mathematics, 21:223-226, 1978.

[48] F. Gavril. Algorithms on circular-arc graphs. Networks, 4:357-369, 1974. 
[49] M. C. Golumbic and R. E. Jamison. The edge intersection graphs of paths in a tree. Journal of Combinatorial Theory, Series B, 38:8-22, 1985.

[50] M. C. Golumbic, H. Kaplan, and R. Shamir. Graph sandwich problems. Journal of Algorithms, 19:449473,1995

[51] J. E. Goodman, R. Pollack, and R. Wenger. Geometric transversal theory. In J. Pach, editor, New Trends in Discrete and Computational Geometry, number 163-198. Springer-Verlag, Berlin, 1993.

[52] M. Groshaus and J. L. Szwarcfiter. Hereditary Helly classes of graphs. Submitted, 2005.

[53] M. Groshaus and J. L. Szwarcfiter. Biclique-Helly graphs. Submitted, 2006.

[54] M. Groshaus and J. L. Szwarcfiter. The biclique matrix of a graph. In preparation, 2006.

[55] R. C. Hamelink. A partial characterization of clique graphs. Journal of Combinatorial Theory, 5:192$197,1968$.

[56] P. Hell. Rétractions de graphes. Ph.D. Thesis, Université de Montreal, 1972.

[57] E. Helly. Ueber mengen konvexer koerper mit gemeinschaftlichen punkter, Jahresber. Math. Verein., 32:175-176, 1923.

[58] R. E. Jamison. Partition numbers for trees and ordered sets. Pacific Journal of Mathematics, 96:115$140,1981$.

[59] F. Larrión, C. P. de Mello, A. Morgana, V. Neumann-Lara, and M. A. Pizaña. The clique operator on cographs and serial graphs. Discrete Mathematics, 282(1-3):183-191, 2004.

[60] F. Larrión and V. Neumann-Lara. A family of clique divergent graphs with linear growth. Graphs and Combinatorics, 13:263-266, 1997.

[61] F. Larrión, V. Neumann-Lara, and M. A. Pizaña. Clique divergent clockwork graphs and partial orders. Discrete Applied Mathematics, 141(1-3):195207, 2004.

[62] F. Larrión, V. Neumann-Lara, and M. A. Pizaña. On expansive graphs. Manuscript, 2005.
[63] F. Larrión, V. Neumann-Lara, and M. A. Pizaña. Graph relations, clique divergence and surface triangulations. Journal of Graph Theory, 51:110-122, 2006.

[64] F. Larrión, V. Neumann-Lara, M. A. Pizaña, and T. D. Porter. A hierarchy of self-clique graphs. Discrete Mathematics, 282(1-3):193-208, 2004.

[65] M. C. Lin and J. L. Szwarcfiter. Characterizations and linear time recognition for Helly circular-arc graphs. In 12th Annual International Computing and Combinatorics Conference, Lecture Notes in Computer Science, to appear, Taipei, Taiwan, 2006.

[66] L. Lovász. Normal hypergraphs and the perfect graph conjecture. Discrete Mathematics, 2:253$267,1972$.

[67] L. Lovász. Combinatorial Problems and Exercises. North-Holland, Amsterdam, 1979.

[68] L. Lovász. Perfect graphs. In R. W. Beineke and R. J. Wilson, editors, Selected Topics in Graph Theory, pages 55-87. Academic Press, New York, N. Y., 1983.

[69] C. L. Lucchesi, C. P. Mello, and J. L. Szwarcfiter. On clique-complete graphs. Discrete Mathematics, 183:247-254, 1998.

[70] T. A. Mckee and F. R. McMorris. Topics in Intersection Graph Theory. SIAM Monographs on Discrete Mathematics and Applications, Philadelphia, PA, 1999.

[71] J. W. Moon and L. Moser. On cliques in graphs. Israel Journal of Mathematics, 3:23-28, 1965.

[72] V. Neumman-Lara. A theory of expansive graphs. Manuscript, 1995

[73] T. Nishizeki and N. Chiba. Planar Graphs: Theory and Algorithms. Annals of Discrete Mathematics 32, North Holland, Amsterdam, New York, Oxford, Tokyo, 1988.

[74] M. C. Paul and S. H. Unger. Minimizing the number of states in incompletely specified sequential switching functions. IRE Transactions Eletronic Computers EC-8, pages 356-367, 1959.

[75] M. A. Pizaña. The icosahedron is clique divergent. Discrete Mathematics, 262(1-3):229-239, 2003. 
[76] E. Prisner. Hereditary clique-Helly graphs. Journal of Combinatorial Mathematics and Combinatorial Computing, 14:216-220, 1993.

[77] E. Prisner. Graph Dynamics. Pitman Research Notes in Mathematics, Longman, 1995.

[78] E. Prisner. Bicliques in graphs I: Bounds on their number. Combinatorica, 20(1):109-117, 2000.

[79] T. M. Przytycka, G. Davis, N. Song, and D. Durand. Graph theoretical insights into evolution of multidomain proteins. In RECOMB 2005 (LNBI, vol. 3500), pages 311-325, 2005.

[80] F. S. Roberts and J. H. Spencer. A characterization of clique graphs. Journal of Combinatorial Theory, Series B, 10:102-108, 1971.

[81] P. J. Slater. A characterization of SOFT hypergraphs. Canadian Mathematical Bulletin, 21:335337, 1978.

[82] J. P. Spinrad. Efficient Graph Representation. American Mathematics Society, Providence, RI, 2003.

[83] J. L. Szwarcfiter. Recognizing clique-Helly graphs. Ars Combinatoria, 45:29-32, 1997.

[84] J. L. Szwarcfiter. A survey on clique graphs. In B. A. Reed and C. L. Sales, editors, Recent Advances in Algorithms and Combinatorics, pages 109-136. Springer-Verlag, New York, N. Y., 2003.

[85] J. L. Szwarcfiter and C. F. Bornstein. Clique graphs of chordal and path graphs. SIAM Journal on Discrete Mathematics, 7(2):331-336, may 1994.

[86] S. Tsukiyama, M. Ide, H. Ariyoshi, and I. Shirakawa. A new algorithm for generating all the maximal independent sets. SIAM Journal on Computing, 6(3):505-517, sep 1977.

[87] Zs. Tuza. Extremal Problems on Graphs and Hypergraphs. PhD Thesis, Acad. Sci., Budapeste, 1983. [Hungarian].

[88] Zs. Tuza. Helly-type hypergraphs and Sperner families. Europ. J. Combinatorics, 5:185-187, 1984.

[89] Zs. Tuza. Helly property in finite set systems. Journal of Combinatorial Theory, Series A, 62:1-14, 1993.

[90] Zs. Tuza. Extremal bi-Helly families. Discrete Mathematics, 213:321-331, 2000.
[91] V. I. Voloshin. On the upper chromatic number of a hypergraph. Australasian Journal of Combinatorics, 11:25-45, 1995.

[92] W. D. Wallis and G.-H. Zhang. On maximal clique irreducible graphs. Journal of Combinatorial Mathematics and Combinatorial Computing, 8:187-193, 1990. 